\title{
BATSE Observations of the Large-Scale Isotropy of Gamma-Ray Bursts
}

\author{
Michael S. Briggs, William S. Paciesas, and Geoffrey N. Pendleton \\ Department of Physics, University of Alabama in Huntsville, Huntsville, AL 35899 \\ Charles A. Meegan, Gerald J. Fishman, John M. Horack, \\ and Martin N. Brock \\ NASA/Marshall Space Flight Center, Huntsville, AL 35812 \\ Chryssa Kouveliotou \\ Universities Space Research Association \\ NASA/Marshall Space Flight Center, Huntsville, AL 35812 \\ Dieter H. Hartmann \\ Department of Physics and Astronomy \\ Clemson University, Clemson, SC 29634 \\ and \\ Jon Hakkila \\ Department of Physics and Astronomy \\ Mankato State University, Mankato, MN 56002
}

To appear in The Astrophysical Journal on 1996 March 1.

(c) 1995 by The American Astronomical Society. All Rights Reserved. 


\begin{abstract}
We use dipole and quadrupole statistics to test the large-scale isotropy of the first 1005 gamma-ray bursts observed by the Burst and Transient Source Experiment (BATSE). In addition to the entire sample of 1005 gamma-ray bursts, many subsets are examined. We use a variety of dipole and quadrupole statistics to search for Galactic and other predicted anisotropies and for anisotropies in a coordinate-system independent manner. We find the gamma-ray burst locations to be consistent with isotropy, e.g., for the total sample the observed Galactic dipole moment $\langle\cos \theta\rangle$ differs from the value predicted for isotropy by $0.9 \sigma$ and the observed Galactic quadrupole moment $\left\langle\sin ^{2} b-\frac{1}{3}\right\rangle$ by $0.3 \sigma$. We estimate for various models the anisotropies that could have been detected. If one-half of the locations were within $86^{\circ}$ of the Galactic center, or within $28^{\circ}$ of the Galactic plane, the ensuing dipole or quadrupole moment would have typically been detected at the $99 \%$ confidence level. We compare the observations with the dipole and quadrupole moments of various Galactic models. Several Galactic gamma-ray bursts models have moments within $2 \sigma$ of the observations; most of the Galactic models proposed to date are no longer in acceptable agreement with the data. Although a spherical Dark Matter Halo distribution could be consistent with the data, the required core radius is larger than the core radius of the Dark Matter Halo used to explain the Galaxy's rotation curve. Gamma-ray bursts are much more isotropic than any observed Galactic population, strongly favoring but not requiring an origin at cosmological distances.
\end{abstract}

Subject headings: gamma-rays: bursts - methods: data analysis - methods: statistical 


\section{Introduction}

The presence of a large-scale pattern in the locations of gamma-ray bursts (GRBs) would be a major clue to their origin. However, the gamma-ray burst locations determined by the Burst and Transient Source Experiment (BATSE) on the Compton Gamma-Ray Observatory (CGRO) remain consistent with large-scale isotropy. This indicates either that the GRBs are isotropic or that their anisotropy is too small to have yet been detected. The upper-limits on the magnitudes of anisotropies strongly constrain Galactic models.

One possible reason for not detecting an anisotropy is that we are observing gamma-ray bursts to a depth much less than the length scale of their distribution. This was thought to be the case prior to BATSE, when it was generally believed that gamma-ray bursts originate from a disk population of neutron stars (Hurley 1986; Higdon \& Lingenfelter 1990; Harding 1994). However, this possibility is excluded by BATSE's concurrent observation that gamma-ray bursts are inhomogeneous, i.e., there is a deficiency of faint GRBs compared to a uniform density in Euclidean space. The inhomogeneity of the GRBs is demonstrated by $\left\langle V / V_{\max }\right\rangle<0.5$ and by the deviation of the $\log (N>P)-\log P$ distribution from a $-3 / 2$ slope power law for the fainter GRBs (Meegan et al. 1992a; Fishman et al. 1994; Meegan 1994a; Horack \& Emslie 1994). The effect is not small: the number of GRBs with peak flux $P=0.8$ photons $\mathrm{s}^{-1} \mathrm{~cm}^{-2}$ between 50 and $300 \mathrm{keV}$ on the $256 \mathrm{~ms}$ timescale is deficient by a factor of about six relative to the extrapolation of the $-3 / 2$ slope power law characteristic of the homogeneous GRBs brighter than 10 photons $\mathrm{s}^{-1} \mathrm{~cm}^{-2}$ (Pendleton et al. 1995). Assuming a galactic origin, the observed inhomogeneity means that instead of viewing to

less than a length scale, BATSE is viewing many length scales and sees past the "edge" of the distribution.

Another possible explanation for not detecting an anisotropy, even though we are seeing past the edge of the distribution, is that our offset from the center of the distribution is very small. A local version of this possibility is that gamma-ray bursts originate from a cometary cloud around the Sun (Bickert \& Greiner 1993; Katz 1993; White 1993; Luchkov 1994). In this hypothesis, the Earth-Sun distance is too small relative to the diameter of the comet cloud to cause a detectable anisotropy and the edge of the comet distribution causes the inhomogeneity. A distant version of this possibility is that gamma-ray bursts originate from a Galactic halo, in which case the length scale of the halo must be large compared to the distance from the Sun to the center of the Galaxy (Fishman 1979; Jennings 1982; Shklovskii \& Mitrofanov 1985; Atteia \& Hurley 1986). Finally, gamma-ray bursts might be isotropic because they originate at cosmological distances (Prilutski \& Usov 1975; Usov \& Chibisov 1975; van den Bergh 1983; Paczyński 1986). In this hypothesis, the observed inhomogeneity is due either to the non-Euclidean geometry of the Universe, to source 
evolution, or to both. If GRBs come from $z \lesssim 1$, too few have been observed to detect the dipole moment due to our motion with respect to distant matter (Brainerd 1995).

Arguably, the most crucial question about gamma-ray bursts is their distance. While discovering or placing stringent limits on large-scale anisotropies promises to disclose the distance scale, discovering gamma-ray burst repeaters would not, since there are cosmological models allowing or predicting repetition (McBreen \& Metcalfe 1988; Dermer \& Schlickeiser 1994; McBreen, Plunkett \& Metcalfe 1993; Brainerd 1994). The discovery of GRB repetition would, however, disprove the most popular model for a cosmological origin, the mergers of compact stars.

The BATSE team has described its findings that gamma-ray bursts are isotropic and inhomogeneous in previous papers and circulars (Meegan et al. 1991; Meegan et al. 1992a; Meegan et al. 1992b; Briggs et al. 1993a; Briggs et al. 1993b; Meegan et al. 1993a; Horack et al. 1993; Fishman et al. 1994; Horack et al. 1994; Meegan et al. 1994a; Briggs et al. 1994). Many authors have emphasized that the isotropic and inhomogeneous GRB spatial distribution observed by BATSE is natural if gamma-ray bursts originate at cosmological distances (Paczyński 1991a; Dermer 1992; Mao \& Paczyński 1992a; Piran 1992; Fenimore et al. 1993; Wickramasinghe et al. 1993; Woods \& Loeb 1994). Paczyński \& Xu (1994) state that "A conservative conclusion is that the sources are at cosmological distances." Other authors have emphasized the possibility of a Galactic origin with an anisotropic pattern too small to have been detected with BATSE, such as a very extended halo distribution or a combination of source distributions (Brainerd 1992; Eichler \& Silk 1992; Hartmann 1992; Li \& Dermer 1992; Lingenfelter \& Higdon 1992; Atteia \& Dezalay 1993; Fabian \& Podsiadlowski 1993; Liang \& Li 1993; Smith \& Lamb 1993; Hartmann et al. 1994a; Higdon \& Lingenfelter 1994; Li, Duncan \& Thompson 1994; Lyne \& Lorimer 1994; Podsiadlowski, Rees \& Ruderman 1995). These authors have generally argued that GRB spectral and temporal properties make a Galactic origin likely. Quashnock \& Lamb (1993a) identified a subset of the first BATSE (1B) catalog (Fishman et al. 1994) which has a concentration towards the Galactic plane and center, a pattern which they interpreted as demonstrating that all gamma-ray bursts originate in the spiral arms of the Milky Way.

In this paper we test the isotropy of the locations of a larger sample, the first 1005 gamma-ray bursts detected by BATSE. Isotropy means that the probability of a GRB occurring in a given region of the sky is solely proportional to the solid angle of that region. While isotropy requires the absence of patterns on all angular scales, herein we emphasize the large angular scale properties of gamma-ray bursts by testing isotropy with dipole and quadrupole tests. We will analyze many subsets, including ones generated by the criteria of Quashnock \& Lamb (1993a). We show that the locations are consistent with isotropy, 
derive upper limits on the parameters of several anisotropic models, and compare the observed moments with those of Galactic models.

The most plausible anisotropy to which dipole and quadrupole tests are insensitive is small-scale clustering, including GRB repetition, which averages to isotropy on large angular scales. Tests sensitive to such anisotropies include those based upon the nearest neighbor distribution and the two-point angular correlation function. Meegan et al. (1995), Hartmann et al. (1994b), and Brainerd et al. (1995) apply these tests and others to the data of the second BATSE catalog (2B) (Meegan et al. 1994b) and find no evidence for small-scale anisotropies in BATSE's GRB locations. While with the $2 \mathrm{~B}$ catalog these authors do not confirm the evidence for GRB repetition found by Quashnock \& Lamb (1993b) and by Wang \& Lingenfelter $(1993 ; 1995)$ in their analyses of the 1B catalog, they do not completely exclude burst repetition: the $99 \%$ confidence upper-limit placed on the repeater fraction by Meegan et al. (1995), Hartmann et al. (1994b), and Brainerd et al. (1995) is $20 \%$.

\section{Dipole and Quadrupole Tests and Distributions}

We test the large-scale isotropy of gamma-ray bursts by calculating various statistics which measure deviations from isotropy and comparing the observed values to the distribution of values expected if GRBs were isotropic. If a statistic is found to have a value highly improbable assuming GRBs to be isotropic, then the null hypothesis of isotropy is statistically contradicted. Several different statistics are used because each is most sensitive to finding certain patterns of anisotropies.

Large angular scale anisotropies are best searched for with dipole and quadrupole statistics. Various dipole and quadrupole statistics have been introduced to the field of gamma-ray burst studies by Hartmann \& Epstein (1989), Paczyński (1990) and Briggs (1993) and are reviewed by Briggs (1993). Dipole statistics are sensitive to a concentration of GRBs towards one direction on the sky while quadrupole statistics are sensitive to concentrations in a plane or towards two opposite poles. Any plausible large-scale concentration should be revealed by its dipole or quadrupole moments before higher moments become significant.

Dipole and quadrupole statistics based upon a particular coordinate system are used since they will be most sensitive to anisotropies in that coordinate system. Galactic-based tests are used because they are most sensitive to Galactic anisotropies, which would result from a Galactic origin for GRBs. Equatorial-based statistics are used because these 
statistics are most sensitive to the artificial anisotropy induced by BATSE's nonuniform sky exposure. We calculate the dipole moment towards the Sun and the quadrupole moment in the ecliptic plane to test for a heliocentric origin. A final pair of dipole and quadrupole statistics are constructed in a coordinate-system independent manner in order to search for anisotropies in unexpected directions in a model-independent manner (Briggs 1993). These statistics are characterized in Table 1.

Each statistic is used as a one-tailed test: the sign of the deviation that is significant is indicated in the last column of Table 1. We use the coordinate-system based tests as one-tailed tests because the models tested, a Galactic origin, a solar system origin, and BATSE's sky exposure, predict the sign of the expected deviation. We use the coordinate-system independent tests $\mathcal{W}$ and $\mathcal{B}$ as one-tailed tests because no random anisotropic distribution can cause small values of these statistics.

We use two probability distributions on the sphere in Monte Carlo simulations designed to test the methods. A standardized form of the Fisher distribution has a dipole moment towards the Galactic center - the probability density per solid angle is proportional to $\exp (\kappa \cos \theta)$, where $\theta$ is the angle between a point and the Galactic center (Fisher et al. 1987). Increasing values of the concentration parameter $\kappa$ result in a greater concentration towards the Galactic center (Figure 1a). A standardized form of the Watson distribution has a Galactic-based quadrupole moment - the probability density per solid angle is proportional to $\exp \left(\kappa \sin ^{2} b\right)$, where $b$ is Galactic latitude (Fisher et al. 1987). Values of the concentration parameter $\kappa<0$ result in a concentration about the Galactic plane, with more negative values of $\kappa$ resulting in greater concentration (Figure $1 \mathrm{~b}-\mathrm{d}$ ). Values of $\kappa>0$ result in a concentration at the Galactic poles. Fisher et al. (1987) and Best \& Fisher (1986) give algorithms for simulating locations from these distributions @.

Figure 2 characterizes these distributions, showing, as a function of $\kappa$, the moments of the distributions. Also shown for the Fisher distribution is the angle $\theta_{\frac{1}{2}}$ such that the region within $\theta_{\frac{1}{2}}$ of the Galactic center contains one-half of the probability. For the Watson distribution, Figure $2 \mathrm{~b}$ shows the angle $b_{\frac{1}{2}}$ such that the region within $b_{\frac{1}{2}}$ of the Galactic plane contains one-half of the probability.

\footnotetext{
${ }^{1}$ Both references have typographic errors in the algorithm for the Watson distribution. The algorithm of Fisher et al. (1987) should have " $\Theta=\arccos S$ " instead of " $\Theta=S$ ". Also note that this algorithm generates locations only for one hemisphere-locations must be moved to the other hemisphere with probability onehalf.
} 


\section{Analysis}

\subsection{Location Determination}

BATSE consists of eight detector modules located at the corners of the Compton Gamma-Ray Observatory. Each module contains a Large Area Detector (LAD) and a Spectroscopy Detector (SD). The results presented here are based upon the LADs which are $50.8 \mathrm{~cm}$ diameter by $1.27 \mathrm{~cm}$ thick $\mathrm{NaI}(\mathrm{Tl})$ scintillators. The instrument triggers and records extensive information whenever there is a $5.5 \sigma$ increase about background in two or more detectors. Further descriptions of the instrument and the various datatypes transmitted are given by Horack (1991) and by Fishman et al. (1994).

BATSE determines GRB locations by comparing the rates in the eight LADs (Brock et al. 1992a; Fishman et al. 1994). The locations are determined by the program LOCBURST by fitting the signal in four detectors and minimizing $\chi^{2}$. The model includes the direct response of the detectors and scattering in the spacecraft and from the earth.

The locations are not precise because of both statistical and systematic limitations. The statistical limitations are due to Poisson fluctuations in the counts observed with the detectors - these errors $\sigma_{\text {stat }}$ are determined by LOCBURST from the derivatives of $\chi^{2}$, which are obtained from the observed counts and the detector model. These errors are well-understood and are Gaussian in the count-regime of BATSE.

Normally there are several datatypes to choose from in order to obtain the best-possible location, but due to the failure of the CGRO tape recorders, for 121 GRBs of our total sample of 1005 GRBs only the MAXBC datatype is available, which consists of the count rates in each detector for the peak one second of emission. Because the background is determined differently for MAXBC data than for other datatypes, until very recently LOCBURST was unable to estimate statistical errors for MAXBC-determined locations.

The statistical errors range from a fraction of a degree for the brightest GRBs to typically $13^{\circ}$ for GRBs at the trigger threshold, as summarized in Table 2. Because the $\sigma_{\text {stat }}$ values were unavailable for MAXBC-located GRBs, these GRBs are not included in Table 2. For bursts in which the MAXBC datatype must be used to determine the location, somewhat larger statistical errors are expected: 1) for bursts longer than $1 \mathrm{~s}$, because of the neglect of source counts outside the peak $1 \mathrm{~s}$ interval, and 2) for bursts shorter than $1 \mathrm{~s}$, because of the reduced SNR caused by including background-only times in the $1 \mathrm{~s}$ long source interval. After most of the analysis of this paper was accomplished, LOCBURST was improved to correctly estimate the statistical errors of MAXBC-determined locations. Based upon the currently available sample of 24 reprocessed MAXBC locations, the 
statistical error distribution of MAXBC-determined locations is only moderately worse than that of locations determined with other datatypes: 14 of the 24 GRBs have $\sigma_{\text {stat }}<3.7^{\circ}$ and only 3 have $\sigma_{\text {stat }}>13.8^{\circ}$, with the largest value being $24^{\circ}$.

The accuracy of BATSE's GRB locations have been verified in several ways. For bright GRBs accurately determined locations are available from the Interplanetary Network (IPN) (Hurley 1993). Comparison of these locations with the locations determined from the LAD rates show that the systematic error of the BATSE locations is currently typically $4^{\circ}$ (Fishman et al. 1994, Hurley et al. 1994). These systematic errors are due to calibration uncertainties, imperfect background subtraction and other approximations in the data analysis. We estimate the $1 \sigma$ location error for a GRB as the root-mean-square sum of its estimated statistical error and a $4^{\circ}$ systematic error. Since the $4^{\circ}$ error is a systematic error, its distribution need not be Gaussian, and we find from comparison of LAD-determined locations with IPN-determined locations that the total error is approximately Gaussian, with somewhat larger 'tails' (Fishman et al. 1994). The excess tails of the distribution are due to the systematic error component and are unimportant for bursts for which the statistical error is dominant.

In addition to their somewhat larger statistical errors $\sigma_{\text {stat }}$, MAXBC-located GRBs should have somewhat larger systematic errors $\sigma_{\text {sys }}$ because the background is determined on-board the spacecraft by a simpler algorithm than that used by LOCBURST on the ground. Koshut et al. (1994) analyzed the MAXBC-determined locations of 33 events with locations determined by the IPN or known from their solar origin and found that the systematic error was $\lesssim 7^{\circ}$.

The locations have also been verified by examining events from sources of known location: solar flares, Cygnus X-1 fluctuations and triggers from SGRs (Fishman et al. 1994; Meegan et al. 1993b; Kouveliotou et al. 1993a; Kouveliotou et al. 1994a) These verifications are important since they are not limited to the bright events required for IPN-locations: solar flares can be of any intensity and Cygnus X-1 fluctuations and SGRs events are near threshold. Fluctuations from Cygnus X-1 are more difficult to locate than GRBs of comparable intensity because they are always short and are superimposed on a background of sub-trigger fluctuations. The distribution of separations between the location of Cygnus X-1 and the LOCBURST-determined locations of the fluctuations has a standard deviation of $13^{\circ}$ (Meegan et al. 1993b), the typical value of $\sigma_{\text {stat }}$ for events near threshold. For these weak triggers, the systematic error $\sigma_{\text {sys }}=4^{\circ}$ is unimportant.

The program LOCBURST has undergone steady improvement: the version used to determine the post-2B locations used herein is slightly better than the version used for the post-1B locations of the $2 \mathrm{~B}$ catalog, which is distinctly better than the version used for $1 \mathrm{~B}$ 
locations. After the calculation of the locations used herein, major improvements have been made to LOCBURST which reduce the systematic error to $\lesssim 2^{\circ}$. As we demonstrate below, dipole and quadrupole moments are relatively insensitive to locations errors so that all of the locations are useful for this analysis. The location improvements are important for tests of clustering or repetition, since the statistical signal rapidly strengthens with improved locations (Brainerd et al. 1995; Hartmann et al. 1994b). At the time of this writing 95 of the locations used herein, all post-2B, have been redetermined with the newest version of LOCBURST. Comparison of these revised locations with the locations used herein show that $50 \%$ of the locations have changed by less than $4.8^{\circ}$ and only $10 \%$ have changed by more than $14.0^{\circ}$. The largest shift is $28^{\circ}$. In terms of the old total error estimates $\sigma_{\text {tot }}=$ $\sqrt{4^{2}+\sigma_{\text {stat }}}, 50 \%$ have moved by less than $0.9 \sigma_{\text {tot }}$ and $90 \%$ have moved by less than $2.7 \sigma_{\text {tot }}$. The worst shift in terms of $\sigma_{\text {tot }}$ is an $18^{\circ}$ change that is $4.6 \sigma_{\text {tot }}$.

The anisotropic response of BATSE's detectors, which enables determination of GRB locations, implies an intensity threshold for detecting a GRB which varies across the sky (Brock 1992b). This intensity-dependent, non-uniform sky response is best described in CGRO coordinates. Because of the many orientations of CGRO for the various observation periods, this effect averages away and need not be further considered.

Because CGRO is in a low-Earth orbit, about one-third of the sky is blocked by the Earth. This causes the equatorial regions to be observed with about $20 \%$ less exposure than the polar regions. Additionally, because burst triggering is disabled during passages through the South Atlantic Anomaly (SAA) and several other regions of enhanced charged particle background, the South Pole receives about 15\% less exposure than the North Pole. Both of these effects are intensity independent and are best described in equatorial coordinates: right ascension, $\alpha$, and declination, $\delta$. Over the spacecraft orbital precession period of about 50 days, the right ascension dependence of the exposure averages away, leaving the sky exposure a function only of declination: $T(\delta)$. The sky exposure $T(\delta)$ has been measured (Fishman et al. 1994), allowing corrections for this effect to be made.

\subsection{Analysis Methods}

The values of the statistics are calculated from the locations; consequently, if many samples are "drawn" from a population, the statistics will have different values for each of the samples. The fluctuations in a finite sample limit the ability of even a perfect instrument to detect a small anisotropy. To demonstrate this effect, Monte Carlo simulations of 250 isotropic locations were made. Figure 3 shows histograms of the values of $\langle\cos \theta\rangle$ and $\left\langle\sin ^{2} \delta-\frac{1}{3}\right\rangle$ obtained from the simulations overlaid with the theoretically expected 
asymptotic distributions (solid curves). As a characterization of the expected fluctuations, Table 1 lists the expected standard deviations $\sigma$ of the six statistics, derived from the asymptotic distributions. The asymptotic distributions may not be sufficiently accurate for small numbers of gamma-ray bursts $N_{B}$ and extreme values of the statistics, which is especially likely for $\left\langle\sin ^{2} b-\frac{1}{3}\right\rangle$ (Briggs 1993). When necessary we use the actual distributions of the statistics as determined by Monte Carlo simulations of isotropy.

An artificial anisotropy should exist in the observed sample of GRB locations because BATSE's sensitivity is not uniform across the sky. The resulting bias expected in the statistics has been been found by Monte Carlo simulations - isotropic locations are created using a random number generator and included in the simulated sample with a probability obtained from the sky exposure map $T(\delta)$. Locations are generated until $N_{B}$ have been accepted and then the values of the statistics are calculated. This effect is illustrated in Figure 3 by the second histograms with their overlying dashed curves. The histograms are still Gaussian, but the artificial anisotropy of the sky exposure has caused the expected mean of the observation to shift from zero. In addition, there is a very small change in the widths of the distributions. The shift in the mean is large for $\left\langle\sin ^{2} \delta-\frac{1}{3}\right\rangle$ because this equatorial-based statistic is sensitive to the time-averaged shadowing of the celestial equator by the Earth. The shift in the mean of $\langle\cos \theta\rangle$ is small because the dipole induced by the equatorial North-South asymmetry is a smaller effect, relative to the standard deviation $\sigma$ of the fluctuations, than the quadrupole moment induced by the Earth blockage and because only part of the equatorial dipole is projected onto the direction towards the Galactic center. The fraction of the artificial dipole moment projected equals the cosine of the angle between the equatorial plane and the Galactic plane, which is $\cos \left(60^{\circ}\right)=1 / 2$.

Table 3 lists the means and standard deviations of the eight statistics expected for isotropy modified by BATSE's actual sky exposure. These sky-exposure-corrected mean values of the statistics have been determined using BATSE's sky exposure accumulated for the time period of the first BATSE catalog (Fishman et al. 1994), the 320 days from 1990 April 21 to 1992 March 5. Work is in progress to measure the sky exposure after this period, a task made difficult by the failure of the CGRO tape recorders. The expected values have also been calculated for the sky exposure map for the first 165.5 days (Brock et al. 1992b) - the differences are insignificant. We expect the changes in BATSE's sky exposure with time to remain small, so the extrapolation of the current sky exposure map to $N_{B}=2000$ gamma-ray bursts should be a good approximation.

These two effects, the finite sample fluctuations and the shifts in the expected means due to BATSE's sky exposure, are illustrated in Figure 4. The dashed lines show the results expected ignoring BATSE's sky exposure, while the solid lines indicate the results 
expected based upon BATSE's sky exposure. The bold lines show the expected means of the statistics while the normal-weight lines show the $\pm 1 \sigma$ envelopes of the distributions of the statistics. The widths of the distributions (indicated by the outer lines) are due to the finite sample fluctuations, while the shifts of the means (indicated by the separation between the dashed, bold lines and the solid, bold lines) are due to the artificial anisotropy induced by BATSE's sky exposure.

The lines on Figure 4 allow visual evaluation of the effects of BATSE's nonuniform sky exposure. We consider the effect of the nonuniform sky exposure to be major when it causes the corrected mean of a statistic to differ from the ideal mean by $\gtrsim 1 \sigma$, i.e., when the bold, solid line is outside of the outer dashed lines. For the Galactic-based statistics, $\langle\cos \theta\rangle$ and $\left\langle\sin ^{2} b-\frac{1}{3}\right\rangle$, this only happens for $N_{B} \gtrsim 2000$. These statistics are relatively insensitive to the nonuniform sky exposure because the Earth's equator is highly inclined to the Galactic equator. The coordinate-system independent quadrupole statistic $\mathcal{B}$ is not importantly affected until $N_{B} \gtrsim 400$, while the coordinate-system independent dipole statistic $\mathcal{W}$ is not importantly affected by the nonuniform sky exposure until $N_{B} \gtrsim 1200$. Because the nonuniform sky exposure originates in the equatorial coordinate system, the equatorial-based statistics are quite sensitive to it: the effects are important for $\langle\sin \delta\rangle$ for $N_{B} \gtrsim 500$ locations and for $\left\langle\sin ^{2} \delta-\frac{1}{3}\right\rangle$ for $N_{B} \gtrsim 130$ locations. The dipole statistic $\langle\sin \delta\rangle$ detects the $\simeq 15 \%$ increased exposure of the North Pole relative to the South Pole, while the quadrupole statistic $\left\langle\sin ^{2} \delta-\frac{1}{3}\right\rangle$ detects the $\simeq 20 \%$ reduced exposure of the equator relative to the poles.

As described above, the locations of the GRBs are uncertain, which propagates into uncertainties in the values of the statistics. Because the location errors are small compared to the scale of large-scale anisotropies, the corresponding errors in the values of the statistics are negligible. Monte Carlo simulations were conducted to demonstrate this. The histograms on the left of each panel of Figure 5 are based upon $5 \times 10^{5}$ simulations of 1000 isotropic locations. The curves overlaying these histograms are the asymptotic distributions of the statistics. The vertical lines on Figure 5 show the critical values of the statistics corresponding to confidence levels against the null hypothesis of isotropy of $99 \%$, $99.9 \%$, and $99.99 \%$. These critical values are defined by the requirement that the chance probabilities under the assumption of isotropy of more extreme values of the statistics are $1 \%, 0.1 \%$, and $0.01 \%$.

Simulations were made of two distributions with moderately detectable anisotropies. The results of the simulations made assuming perfect determinations of the locations are shown in the bold, right-most histograms on Figure 5. The fractions of these histograms to the right of the vertical lines are the fractions of the simulations in which the anisotropies 
are detected at the confidence levels of the lines. The anisotropic simulations were repeated, this time moving each simulated location an angle $\Sigma$ in a random direction, obtaining the middle histograms of Figure 5.

The angles $\Sigma$ and the fractions of the simulated bursts moved by each particular value of $\Sigma$ are listed in the last two columns of Table 2. The simulated smearing exaggerates with a very substantial safety margin the actual location uncertainties: 1) the values $\Sigma$ are based upon the worst $\sigma_{\text {tot }}$ in each row, 2) the values $\Sigma$ are at least twice this worst location error, 3) the simulated smearing locations are each moved by an angle $\Sigma$, not by an angle selected from a Gaussian distribution of this width, and 4) to allow for outliers additional simulated locations are placed into the category with the largest smearing angle.

Figures $5 \mathrm{a} \& \mathrm{~b}$ show simulations of the Fisher distribution with $\kappa=0.2$, a slightly more concentrated distribution than the example shown in Figure 1a. This distribution places one-half of the probability within $84.3^{\circ}$ of the Galactic center and has an expected dipole moment of $\langle\cos \theta\rangle=0.06649$. The middle histograms, which show the values of the statistics when the locations are smeared, are only slightly shifted from the bold histograms on the right, which show the results for perfect locations. Because the scale of the anisotropy is of order $90^{\circ}$, the smearing by tens of degrees is a small effect. To test the effect of the location errors for a quadrupole distribution, simulations of the Watson distribution for $\kappa=-10$ (Figure 1d) were made - this distribution has one-half of the probability within $|b| \leq 8.7^{\circ}$ and an expected quadrupole moment of $\left\langle\sin ^{2} b-\frac{1}{3}\right\rangle=-0.2833$. This distribution is extremely detectable, so its detectability was reduced by diluting it with isotropic locations, creating anisotropic locations with probability 10\%. In this example (Figures 5c \& 5d), the middle histograms based upon the smeared locations are quite noticeably shifted from the bold histograms based upon the perfect locations, but the smearing has not caused the middle histograms to be similar to the histograms for isotropic locations.

The sensitivity or power of a statistic is the probability of its finding an actual anisotropy (Eadie et al. 1971; Martin 1971). In our case, the powers of the statistics for finding the example anisotropies at the confidence levels of $99 \%, 99.9 \%$ and $99.99 \%$ is given by the fraction of simulations with statistics more extreme than the corresponding critical values, i.e., to the right of the vertical lines in Figure 5. The powers of the statistics are listed in Table 4. The decrease in the fraction of the anisotropic simulations, smeared versus unsmeared, that are past the critical values represents the loss in sensitivity or power to the anisotropy caused by the smearing. Table $4 \mathrm{~A}$ shows that the loss of power for the Fisher $\kappa=0.2$ model is minor. Similar results were obtained by Horack et al. (1993) via simulations of a halo distribution. The model with $90 \%$ isotropic locations and $10 \%$ locations from a narrow disk model was intended to be an extreme case-half of the disk 
probability is contained within $|b| \leq 8.7^{\circ}$ versus the exaggerated smearing angles $\Sigma$ listed in Table 2. Table $4 \mathrm{~B}$ shows that while the smearing substantially reduces the sensitivity to this model anisotropy, the ability to detect the narrow disk component is not eliminated.

The above simulations were each of 1000 locations. We now discuss how our ability to find anisotropies with BATSE depends on the number of gamma-ray bursts $N_{B}$ observed. The finite-sample fluctuations of the coordinate-system based statistics, such as $\langle\cos \theta\rangle$ and $\left\langle\sin ^{2} b-\frac{1}{3}\right\rangle$, asymptotically have a $1 / \sqrt{N_{B}}$ dependence (Tables $1 \& 3$ ) because of the Central Limit Theorem (Paczyński 1990; Briggs 1993). Monte Carlo simulations show that the asymptotic distributions are sufficiently accurate for the values of $N_{B}$ under consideration (Briggs 1993). The coordinate-system independent Rayleigh-Watson $\mathcal{W}$ and Bingham $\mathcal{B}$ statistics are proportional to the product of $N_{B}$ and the moment per GRB squared (Bingham 1974; Watson 1983; Briggs 1993) and their asymptotic distributions are also sufficiently accurate (Briggs 1993). Thus for both types of statistics the finite sample fluctuations cause the magnitude of detectable anisotropic moments to scale as $1 / \sqrt{N_{B}}$. The effects of the location uncertainties scale in the same manner. It doesn't matter where a variance comes from, only that it is finite, in which case the Law of Large Numbers or the Central Limit Theorem tells us that the mean of the measurements will be determined with uncertainty scaling as $1 / \sqrt{N_{B}}$ for large $N_{B}$ (Eadie et al. 1971; Martin 1971; Lyons 1986). This is the well-known result that the knowledge of the mean of a series of numbers with errors improves as $1 / \sqrt{N}$.

As a demonstration that BATSE's ability to find anisotropies improves as $1 / \sqrt{N_{B}}$, $5 \times 10^{4}$ simulations of $10^{4}$ locations were made (Tables $4 \mathrm{C}$ and $4 \mathrm{D}$ ). The simulations in Tables $4 \mathrm{C}$ and $4 \mathrm{D}$ have 10 times the number of locations as those of Tables $4 \mathrm{~A}$ and $4 \mathrm{~B}$, but the moments have been reduced by a factor of $\sqrt{10}$. Allowing for the fluctuations expected in $5 \times 10^{4}$ simulations, the anisotropies are detected in the same fraction of simulations in Tables $4 \mathrm{C}$ and $4 \mathrm{D}$ as in Tables $4 \mathrm{~A}$ and $4 \mathrm{~B}$, confirming the $1 / \sqrt{N_{B}}$ improvement in sensitivity.

Smearing by angles $\Sigma$ as listed in Table 2 reduces the dipole moment $\langle\cos \theta\rangle$ of dipole distributions by a factor of 0.86 and the quadrupole moment $\left\langle\sin ^{2} b-\frac{1}{3}\right\rangle$ of quadrupole distributions by a factor of 0.68 . We found this to be true not only of the distributions listed in Table 4, but also for all the other distributions we simulated. These reduction factors greatly overestimate the effects of BATSE's location uncertainties since the angles $\Sigma$ greatly exaggerate the location uncertainties.

Another possible error in the values of the statistics is due to the possibility of trigger misclassifications. We estimate both the number of GRBs misclassified as non-GRBs and the number of non-GRBs misclassified as GRBs to be about 1\% (Mallozzi et al. 1993; 
Fishman et al. 1994). The estimated misclassifications are roughly equally split between Cygnus X-1 fluctuations and solar flares, so the misclassifications are not all at the same location. We also estimate that at most one Soft Gamma-Ray Repeater (SGR) has been misidentified as a GRB and vice versa (Kouveliotou 1994b). Even if all 9 SGR triggers are added to the set of 1005 gamma-ray GRBs which we analyze, the set remains consistent with isotropy. In an extreme example, if a false population of $2 \%$ of the GRBs is placed at the Galactic Center, a dipole moment of $\langle\cos \theta\rangle=0.02$ is produced, which would be detectable only at the $1.1 \sigma$ level in a sample of 1000 locations.

In summary, the dominant limitation for BATSE in discovering a large-scale anisotropy is the fluctuations resulting from the finite number of GRBs observed, a limitation that no instrument can avoid. The effects of the location uncertainties are negligible and will remain so since the effects of both the finite-sample fluctuations and the location uncertainties scale as $1 / \sqrt{N_{B}}$. The estimated rate of trigger misclassifications is too low to significantly effect the results. The bias induced by BATSE's nonuniform sky exposure can be measured and corrected for when comparing model predictions with the observations.

\section{Results}

The first 1005 gamma-ray bursts observed by BATSE are shown in Figure 6. These GRBs occurred between the enabling of burst triggering on 1991 April 21 and the end of CGRO Viewing Period \#222 on 1994 May 31. The post-2B catalog locations are preliminary, but as discussed in $\S 3.1$, the revised locations will be close to the current locations and are adequate for the study of the large-angular scale properties of gamma-ray bursts. The 585 locations of the second BATSE catalog (2B) are publicly available (Meegan et al. 1994b). The first 262 triggers of the $2 \mathrm{~B}$ catalog are referred to as the revised $1 \mathrm{~B}$ catalog - these differ from the original 1B catalog (Fishman et al. 1994) by the revised classifications of several triggers.

The values of the six statistics for the first 40, 100, 262 (=1B revised), $585(=2 \mathrm{~B})$ and 1005 gamma-ray bursts observed by BATSE are shown on Figure 4 with dots (note that these sets are not independent since each is a subset of the latter sets). A greater than $1 \sigma$ deviation of a statistic from the value expected for isotropy is indicated by the corresponding dot lying outside of the outer solid lines, which represent the $\pm 1 \sigma$ envelopes of the distributions of the statistics, including the effects of BATSE's sky exposure. This occurs several times, but only by a small margin. Thus the depicted statistics are all consistent with isotropy. 
In order to test BATSE's sky exposure map, Table 5 presents the Earth-based statistics, $\langle\sin \delta\rangle$ and $\left\langle\sin ^{2} \delta-\frac{1}{3}\right\rangle$, for the revised 1B catalog, the $2 \mathrm{~B}$ catalog and the first 1005 GRBs. The largest deviation from the predictions based upon the sky exposure map is $1.3 \sigma$; the largest deviation for the first $1005 \mathrm{GRBs}$ is only $0.7 \sigma$. In contrast, the value of $\left\langle\sin ^{2} \delta-\frac{1}{3}\right\rangle$ for the 1005 GRBs is $2.0 \sigma$ away from the mean that would be expected for a uniform sky exposure. This statistic is the most sensitive to BATSE's nonuniform sky exposure and appears to have detected that effect at modest significance. These results are good indications that we are accurately predicting the effects of BATSE's nonuniform sky exposure.

Table 6 lists the subsets into which the set of 1005 GRBs have been split in order to test for possibly anisotropic subsets. Many of these sets have common members and are thus not independent. For each of these sets the Galactic and coordinate-system independent statistics have been calculated and are listed in Table 7.

Because Table 7 lists the values of four statistics for 48 subsets of the 1005 GRBs, it is likely that at least one value of a statistic will have a large deviation from the predictions of the null hypothesis of isotropy. The four statistics are not completely independent, just as the various subsets are not all independent. Ultimately the degree of belief corresponding to a confidence level is a scientific judgement. In our judgement, for the entire dataset an improbability of $\lesssim 0.01$ would be interesting and $\lesssim 0.001$ would be convincing. Because of the many subsets examined, we would decrease these thresholds by at least a factor of 10 when considering a candidate anisotropy present only in a subset.

Of the 48 datasets analyzed in Table 7 , only one deviates from isotropy at more then the $99 \%$ confidence level, namely dataset \#46, the Medium GRBs of the revised 1B catalog. These 51 GRBs are "Medium" according to the selection criteria of Quashnock \& Lamb (1993a): $\log _{10} V<-0.8$ and $465<B<1169$, where $\mathrm{B}$ is a measure of GRB brightness on the $1024 \mathrm{~ms}$ timescale and V compares the peak counts rates on the $64 \mathrm{~ms}$ and 1024 ms timescales (Lamb, Graziani \& Smith 1993). This dataset deviates from isotropy by $+2.4 \sigma$ for $\langle\cos \theta\rangle$ and by $-2.6 \sigma$ for $\left\langle\sin ^{2} b-\frac{1}{3}\right\rangle$. These deviations are in the direction of a concentration towards the Galactic center and the Galactic plane. The dataset of Quashnock \& Lamb (1993a) was selected from the 1B catalog and had four additional GRBs; the $C_{\max } / C_{\min }$ values of these four GRBs on untriggered timescales have been withdrawn by the BATSE team as being erroneous and these four GRBs are therefore not included in the Medium bursts of the revised 1B catalog. The 55 GRB dataset of Quashnock \& Lamb (1993a) deviates from the predictions of isotropy and BATSE's sky exposure map by $+3.2 \sigma$ for $\langle\cos \theta\rangle$ and by $-2.9 \sigma$ for $\left\langle\sin ^{2} b-\frac{1}{3}\right\rangle$. The 51 Medium GRBs of the revised $1 \mathrm{~B}$ catalog are shown with filled circles on Figure 7 while the additional four 
from the original 1B catalog are shown with open circles.

The dipole and quadrupole moments of the Quashnock \& Lamb 55 GRB dataset each have significances of roughly $10^{-3}$. Quashnock \& Lamb point out that these two statistics are partially independent and find from Monte Carlo simulations that the probability of seeing both deviations or larger is $1.9 \times 10^{-6}$. Because they optimized the brightness $\mathrm{B}$ selection criterion to obtain this result, they estimate the probability as $1.1 \times 10^{-4}$. They interpreted their dataset (filled and open circles on Figure 7) as evidence for spiral arms and concluded that all gamma-ray bursts are Galactic in origin (Quashnock \& Lamb 1993a).

The estimation of the correction factor to apply to significances obtained from retrospective analysis of data is difficult. How many ways were the data subdivided? How many statistical approaches were tried? In this case, we should have some additional factor due to the $\mathrm{V}$ selection criterion and to the idea of using a joint probability from $\langle\cos \theta\rangle$ and $\left\langle\sin ^{2} b-\frac{1}{3}\right\rangle$. While retrospective analysis has potential pitfalls, it is necessary because of our limited understanding of gamma-ray bursts.

The best solution is to collect more data in order to make an independent test of a hypothesis formed by retrospective analysis. Using exactly the same selection criteria as Quashnock \& Lamb (Graziani 1993), we find 76 additional Medium GRBs in the first 1005 GRBs - these form our dataset \#47 and are shown with stars on Figure 7. The rate of discovering Medium GRBs has decreased due to data gaps caused by the failure of the CGRO tape recorders which prevent our determining the B and V values for many GRBs. Even a small data gap, which does not hinder GRB localization, prevents determination of the peak count rate because the peak rate may have occurred during the data gap. The data gaps should not have any correlation with intrinsic properties of gamma-ray bursts and thus should not bias the selection. The new Medium GRBs are consistent with isotropy and in fact the $-1.7 \sigma$ deviation of $\langle\cos \theta\rangle$ indicates a deficiency of GRBs towards the Galactic center. The evidence for a Galactic origin of GRBs is not confirmed by a larger, independent sample. The entire dataset of $51+76=127$ Medium GRBs, dataset \#48, is also consistent with isotropy: $\langle\cos \theta\rangle$ deviates by $+0.2 \sigma$ and $\left\langle\sin ^{2} b-\frac{1}{3}\right\rangle$ by $-1.8 \sigma$.

What can we conclude from the contradictory results of datasets \#46 and 47? If one takes dataset \#46 to be evidence of a true anisotropy and thus the values of $\langle\cos \theta\rangle$ and $\left\langle\sin ^{2} b-\frac{1}{3}\right\rangle$ observed for this set to be measurements of true anisotropic moments, then one has the difficulty of explaining the $3.0 \sigma$ and and $1.8 \sigma$ deviations of the moments observed for dataset \#47 from these values. Conversely, if one assumes the GRBs to be isotropic, then one has the difficulty of explaining the $+2.4 \sigma$ and the $-2.6 \sigma$ of deviations of $\langle\cos \theta\rangle$ and $\left\langle\sin ^{2} b-\frac{1}{3}\right\rangle$ for dataset \#46 from the values expected for isotropy. Because dataset \#46 was identified by retrospective analysis and its result is not confirmed by a larger, 
independent sample, we judge that the evidence against the null hypothesis of isotropy is weak.

We use the statistics of Horack et al. (1994) to search for a heliocentric pattern in the first 1005 GRBs. The dipole moment towards the Sun, $\langle\cos \phi\rangle$, is observed to be 0.006 , which deviates by $+0.3 \sigma$ from the value of 0.000 predicted by isotropy and BATSE's sky exposure. Because the direction to the Sun changes with time, the sky exposure bias is calculated by averaging the time-dependent bias specified in Table 3 over 3.1 years. The quadrupole moment in the ecliptic plane, $\left\langle\sin ^{2} \beta-\frac{1}{3}\right\rangle$, is observed to be $0.022, \mathrm{a}+0.2 \sigma$ deviation from the value of 0.020 predicted by isotropy and BATSE's sky exposure. No evidence for a heliocentric origin is found.

We have searched for an excess or deficiency of GRBs near the Magellanic Clouds, the Virgo cluster, M31, the Galactic Center, and $\alpha$ Centauri. In all 48 datasets there is no instance in which the number of GRBs within $20^{\circ}$ of these objects is less probable than $1 \%$.

In summary, we have examined 48 subsets of the first 1005 gamma-ray bursts observed by BATSE using four dipole and quadrupole tests. The entire set of 1005 GRBs has been tested with four additional dipole and quadrupole statistics. Only one set, \#46, appears anisotropic, however, a larger, independent set selected with the same criteria, \#47, fails to confirm the anisotropy so we judge the data to be consistent with isotropy. The largest anisotropy in any set other than \#46 is the $2.1 \sigma$ deviation of $\langle\cos \theta\rangle$ for dataset \#27. Because of the large number of partially independent sets and statistics examined, a deviation of this magnitude is expected. The gamma-ray bursts locations are consistent with large-scale isotropy.

\section{Model Comparisons and Limits}

While the statistics show that gamma-ray burst locations are consistent with isotropy, they do not prove that gamma-ray bursts are isotropic - one could imagine that a small anisotropy exists that cannot be detected in a sample of 1005 locations. This raises the question of how large an anisotropy could exist and not have been detected. We address this question in two ways: First, we discuss below and summarize in Table 8 the comparisons of the moments observed by BATSE with the moments of published Galactic models. Secondly, we use Monte Carlo simulations to determine for a variety of models which anisotropies could have been detected. These upper-limits on anisotropies are discussed below and summarized in Figure 8.

For both the model comparisons (Table 8) and model parameter limits (Figure 8) we 
have assumed that the number of sources is the same as the number of observed bursts. If some of the sources repeat so that there are fewer sources than observed bursts, the finite sample fluctuations will be larger than we have calculated, which will reduce our ability to detect anisotropies (Quashnock 1995). We have placed a 20\% upper-limit on the repeater fraction at the 99\% confidence level (Meegan et al. 1995), which implies that there are at

least 9 sources for each 10 observed bursts. In this case the finite sample fluctuations would be $10 \%$ larger than we have calculated.

\subsection{Model Comparison Methods}

Below and in Table 8 we compare the moments of published Galactic models with the observations. Because the goal of these comparisons is to confront the models with BATSE's observations, the comparisons are made between the observed moments corrected for the sky exposure bias and the model moments. These published models were developed based upon earlier and smaller datasets - some could have improved agreement with the current observations by reoptimizing their parameters. In other cases, full reoptimization might increase the deviations in the moments because of the tighter constraints of the brightness distribution data. Some of the models are merely examples of good fits to older data, rather than best-fits to that data. These models might be improved to have reduced moments, possibly at the expense of less "reasonable" parameters. The uncertainties in the theoretical models are difficult to quantify. In any case, reoptimizing the models is beyond the scope of this paper, so we compare the observations with the published moments without any corrections. We convert the differences between the observed and published model moments to deviations in units of $\sigma$, using for $\sigma$ the finite sample fluctuations of the observations, ignoring the possibility of uncertainties in the models.

The values $\sigma$ are calculated as the sample fluctuations expected assuming isotropy (Table 3) - a more accurate procedure would be to calculate the values of $\sigma$ from the anisotropic models (Li 1995). For models with moments so small that $\sim 1000$ bursts are needed to detect the moment, the difference between the model and isotropy $\sigma$ values are typically $\lesssim 1 \%$. For the model of $\mathrm{Li}$ et al. (1994), which predicts a larger moment in a subset of the data, the difference somewhat exceeds $10 \%$ (Li 1995). Since the differences between the isotropy and model $\sigma$ values are small and the model $\sigma$ values generally have not been published, we calculate the $\sigma$ values assuming isotropy.

In $§ 3.2$ we found that smearing the locations by the angles $\Sigma$ listed in Table 2 reduced the dipole moment $\langle\cos \theta\rangle$ by the factor 0.86 and the quadrupole moment $\left\langle\sin ^{2} b-\frac{1}{3}\right\rangle$ by 0.68. These factors considerably exaggerate the effects of smearing since the angles $\Sigma$ are 
chosen to be at least twice $\sigma_{\text {tot }}$ for the least well-located GRB in each of four categories (Table 2). Simulations using more accurate smearing angles show the smearing factors to be only a few percent below one, and we have therefore neglected this effect in our model comparisons (Table 8).

\subsection{Model-Limit Determination Methods}

Monte Carlo simulations are used to determine for various models, as a function of the number of bursts $N_{B}$, the parameter values that should create detectable anisotropies. The goal of the simulations is to determine what anisotropies should be detectable and therefore the upper-limits are not based upon the moments observed by BATSE.

In order to calculate the ability of a statistic to detect an anisotropy, the "power" of a statistic, it is necessary to assume a particular anisotropic model (Eadie et al. 1971; Martin 1971). For example, the statistic $\langle\cos \theta\rangle$ is highly sensitive to a dipole moment towards the Galactic center but completely insensitive to a dipole moment towards the North Galactic Pole. For each model we determine the detectability of anisotropies for three confidence levels, $99 \%, 99.9 \%$ and $99.99 \%$, which are equivalent to $2.3 \sigma, 3.1 \sigma$, and $3.7 \sigma$ for Gaussian-distributed statistics. The critical values of the statistics are determined from the distribution of the statistics under isotropy so that the chance probability of mistakenly deciding that an isotropic distribution is anisotropic is $1 \%, 0.1 \%$, and $0.01 \%$ (see the vertical lines on Figure 5). Choosing a high confidence level reduces the chance of falsely discovering an anisotropy, a mistake known as a Type I error, at the expense of reducing the ability to discover a true anisotropy (Eadie et al. 1971; Martin 1971). We show the three confidence levels to allow readers to choose based upon their judgement and considerations such as whether the entire dataset or a subset is being tested.

Just as an isotropic distribution may by chance look rather anisotropic, so may an anisotropic distribution appear rather isotropic. This is seen in Figure 5 in the areas of the anisotropic histograms that extend near the center of the isotropic histograms. A Type II error is failing to discover an anisotropy when it actually exists and the power of a statistic is one minus the probability of making a Type II error, i.e., the probability of detecting the anisotropy (Eadie et al. 1971; Martin 1971). We choose to determine the detectability of anisotropies for two powers: for BATSE "typically" and "nearly always" discovering an anisotropy, by which we mean finding the anisotropy at or past the stated confidence level in $50 \%$ and $95 \%$, respectively, of the simulations.

Figure 8 shows as a function of the number of gamma-ray bursts $N_{B}$ the values of the 
anisotropy parameters such that either $50 \%$ or $95 \%$ of the simulations find the anisotropy at one of our confidence levels. The detectabilities are determined as a function of $N_{B}$ for $N_{B}=100$ to 2000 . This allows one to determine what anisotropy could be found in a subset of the current 1005 GRBs or will be detectable in the future. Each panel of Figure 8 thus has six curves: three confidence levels at two different powers. The "typical" detection curves are solid while the "nearly always" curves are dashed. The y-axes are arranged so that more anisotropic models are at the top of the graph, hence, the top curves are for the 99.99\% confidence level and the bottom curves are for the $99 \%$ confidence level.

We note that in all cases detecting an anisotropy "nearly always" at the $99 \%$ confidence level (lowest dashed curves) requires a larger anisotropy than detecting the anisotropy "typically" at the $99.99 \%$ confidence level (highest solid curve). This is an indication of how conservative in the sense of minimizing BATSE's ability to find anisotropies the "nearly always" requirement is.

The detectability of each model is determined for the most sensitive Galactic statistic for that model (left panels) and for the most sensitive coordinate-system independent statistic (right panels). This allows one to determine what Galactic anisotropies and what anisotropies in less-expected directions, e.g., M31, Virgo cluster, Magellanic Clouds, BATSE could detect. With one exception discussed below (\$5.4), the Galactic-based statistic are more sensitive for discovering our example models, which are constructed in Galactic coordinates.

The location errors slightly "isotropize" the observations. The Monte Carlo simulations used to determine the detectability of the model anisotropies (Figure 8) include a "smearing" of the simulated locations by an angle $\sigma=14^{\circ}$. The value $14^{\circ}$ is the root-mean-square sum of the $13^{\circ}$ statistical and $4^{\circ}$ systematic error typical for BATSE's faint GRBs and is therefore conservative in the sense of underestimating our ability to find anisotropies.

\subsection{Fisher and Watson Models}

We use the Fisher and Watson distributions as "generic" dipole and quadrupole distributions to derive some illustrative limits. We assume that all locations generated in the simulations of these distributions are "observed". Figures 8a and 8b show the detectable

values of the concentration parameter $\kappa$ for these two models. Values of $\kappa$ may be converted into dipole and quadrupole moments and into angles containing one-half of the probability using Figure 2.

The lowest curve of the left box of Figure 8a shows that for 1000 GRBs BATSE would 
"typically", i.e., $50 \%$ of the time, detect a Fisher distribution with $\kappa \geq 0.13$ at the $99 \%$ confidence level or higher; a simulated example of this distribution is shown in Figure 1a. The dipole moment $\langle\cos \theta\rangle$ of this distribution is 0.043 , implying an expected significance for 1000 GRBs of $2.4 \sigma$. The Fisher distribution with $\kappa=0.13$ has one-half of the probability within $\theta_{\frac{1}{2}}=86^{\circ}$ of the Galactic center compared to $90^{\circ}$ for isotropy.

A very conservative upper-limit obtained from the middle dashed curve of the left box of Figure 8a is that BATSE would with 1000 locations "nearly always", i.e., $95 \%$ of the time, detect a Fisher distribution about the Galactic center with $\kappa \geq 0.27$ at the $99.9 \%$ confidence level or better. This distribution has one-half of the probability within $\theta_{\frac{1}{2}}=82^{\circ}$ of the Galactic center and an expected moment $\langle\cos \theta\rangle=0.090$, implying a typical detection for 1000 GRBs at the very high level of $4.9 \sigma$.

Similar results for finding a quadrupole moment created by the Watson distribution are given in Figure 8b. A value of $\kappa=-0.28$ would typically be discovered in 1000 locations by the $\left\langle\sin ^{2} b-\frac{1}{3}\right\rangle$ test at the $99 \%$ confidence level (see Figure $1 b$ ). This distribution has one-half of the probability within $b_{\frac{1}{2}}=28^{\circ}$ of the Galactic plane versus $30^{\circ}$ for isotropy. The fact that a plane concentration this weak is detectable but not detected shows how isotropic the GRBs are. The middle dashed curve of the left box of Figure 8b gives very conservative upper limits, e. g., for 1000 GRBs, BATSE would nearly always find at the $99.9 \%$ confidence level a Watson distribution with $\kappa=-0.57$. This distribution has one-half of the probability $26^{\circ}$ of the Galactic plane.

The right box of Figure 8a gives results for detecting a concentration about any point on the sky: a value of $\kappa \geq 0.18$ would be typically detected in 1000 GRBs by the Rayleigh-Watson dipole statistic $\mathcal{W}$ at the $99 \%$ confidence level. The corresponding concentration is having one-half of the probability within $85^{\circ}$ of any point. Similarly the right box of Figure 8b gives results for detecting a concentration about any plane on the sky: a value of $\kappa \leq-0.4$ would be typically detected in 1000 GRBs by the Bingham quadrupole statistic $\mathcal{B}$ at the $99 \%$ confidence level. The corresponding concentration is having one-half of the probability within $27^{\circ}$ of some plane. These results constrain suggestions that gamma-ray bursts originate from the Magellanic Clouds (Maoz 1993a; Fabian \& Podsiadlowski 1993) or from the Milky Way and M31 (Gurevich et al. 1994) or from any other unexpected direction on the sky. 


\subsection{Disk and Spiral Arm Models}

The Watson distribution discussed above serves as a non-physical model of a disk distribution. We also consider a somewhat more physical model: the GRBs are assumed to be standard candles distributed exponentially in distance from the Galactic plane and the solar system is assumed to lie in the plane. Note that the vertical profile of the thin stellar disk of the Milky Way is adequately modeled by an exponential (Gilmore, King \& van der Kruit 1990). The appearance of this distribution is parameterized by the ratio observing depth, which is the maximum distance to which GRBs can be seen, divided by the exponential scale height. For 1000 GRBs, even using the very conservative limit of nearly always detecting the anisotropy at the $99.9 \%$ confidence level, the middle dashed curve of Figure $8 \mathrm{c}$ left shows that BATSE cannot be viewing past 0.8 exponential scale heights. In this model BATSE is viewing a spherical region no larger than 0.8 scale heights and the ratio of the lowest density of GRBs viewed over the highest density is $\exp (-0.8)=0.45$, which is reached only at the North and South Galactic Poles of the spherical region. Since the faint GRBs are actually deficient by a factor of about six, this model clearly does not agree with the inhomogeneous radial distribution observed by BATSE.

As a very simple spiral arm model we consider an infinitely-long cylinder containing a uniform density of standard candle sources, with the Sun located on the cylinder axis. This distribution is parameterized by the ratio observing depth divided by arm radius. Using the very conservative upper limit of nearly always detecting the anisotropy at the $99.9 \%$ confidence level, the middle dashed curve of Figure 8d right shows that the observing depth cannot exceed 1.2 arm radii. The fractional volume of the sphere in which GRBs could be seen occupied by the uniform density cylinder is $83 \%$. Again, this clearly does not match the major deficiency of faint GRBs observed by BATSE.

Only in the spiral arm model is the coordinate-system independent statistic more sensitive than the Galactic-based statistic. This is because $\left\langle\sin ^{2} b-\frac{1}{3}\right\rangle$ is only detecting the concentration the arm makes in the plane, while the Bingham statistic $\mathcal{B}$ is also detecting the concentration of GRBs at the two poles of the cylinder axis. We could construct a special Galactic-based statistic to look for this latter effect, but the limits obtained with $\mathcal{B}$ amply suffice.

In both the disk and spiral arm models one would expect the faintest sources to show the largest anisotropy, but no anisotropy is detected in these GRBs, e.g., datasets \#21, 28, 35 .

It should be clear that a disk or spiral arm model requires a strong anisotropy because of the major deficiency of faint GRBs seen by BATSE. For example, in a disk model, the 
many faint GRBs which are "missing" above and below the plane in order to create the observed inhomogeneity would necessarily produce a concentration in the plane and a large

quadrupole moment. This was shown to be the case for several disk models by Paczyński (1990, 1991b) and has been confirmed for a variety of models by Mao and Paczyński (1992b) and Hakkila et al. (1994a). The inability of spiral arm models to fit the BATSE data has been shown by Hakkila et al. (1994a) and by Smith (1994a). A model with more than one spiral arm visible would be subject to the constraints against an excess in the plane characteristic of disk models.

\subsection{Geometric Halo Models}

The basic requirements on a halo model are: 1) the length scale of the distribution should be $\gg$ the solar galactocentric distance $R_{\circ}=8.5 \mathrm{kpc}$, so that the distribution will appear sufficiently isotropic, 2) the observing depth (the maximum distance seen by BATSE) should be $\gg$ the length scale of the distribution so that there will be a sufficient deficiency of faint GRBs, i.e., inhomogeneity, and 3) the observing depth should be $\lesssim 350$ $\mathrm{kpc}$, half the distance to M31, so that the model will not predict an excess of GRBs from M31. Since the focus of this paper is large-scale isotropy, we will primarily be concerned with the first requirement: that our offset from the center of the galaxy be small compared to the size of the observed GRB distribution so that the dipole moment not be too large. Generally the constraint imposed by the quadrupole moment is less limiting.

Any spherically symmetric halo model may be thought of as the sum of a series of shells. Hartmann et al. (1994a) have shown that a shell of galactocentric radius $\mathrm{R}$ has a dipole moment

$$
\langle\cos \theta\rangle=\frac{2}{3} \frac{R_{\circ}}{R} .
$$

Thus a shell of radius $R=110 \mathrm{kpc}$ has a dipole moment $2 \sigma$ above the value observed for 1005 GRBs (corrected for the sky exposure bias). Any GRBs interior to this radius will have to be balanced by GRBs exterior to this radius.

As our simplest halo model we consider a sphere centered on the Galactic center containing a uniform density of sources, all of which are observable by BATSE. Wasserman (1992) has considered a similar model. The lowest solid curve of the left panel of Figure 8e shows that the radius $R_{\text {halo }}$ of this sphere must be greater than $194 \mathrm{kpc}$ to avoid BATSE typically detecting the anisotropy in 1005 GRBs at the $99 \%$ confidence level.

Our next model is based upon a simple functional form for the Dark Matter Halo used 
to explain the rotation curves of galaxies including the Milky Way: density

$$
\rho(R) \propto \frac{1}{1+\left(R / R_{\text {core }}\right)^{2}}
$$

The $R^{-2}$ dependence yields the observed flat rotation curves and the core radius prevents an excess contribution at the center and prevents the function from diverging. In contrast, the distribution of the luminous halo of our Galaxy is at least as steep as $R^{-3.5}$ (Gilmore, King \& van der Kruit 1990; Djorgovski \& Meylan 1994) which is more anisotropic than the $R^{-2}$ distribution that we use. Hakkila et al. (1994b) found using 452 GRBs that exponents from 1.5 to 4.0 were acceptable. Paczyński (1991b) and Mao \& Paczyński (1992b) used this distribution and concluded that there would be an observable deficiency of faint sources and an undetectable dipole moment only if $R_{\text {core }}>2 R_{\circ}=17 \mathrm{kpc}$. Brainerd (1992) found that a somewhat smaller value of $R_{\text {core }}$ was permitted if GRBs are not standard candles.

Figure 8f presents our results for a Dark Matter Halo of standard candle sources observed to $300 \mathrm{kpc}$. This observing depth is near the maximum permitted without seeing sources from M31 and is near the best value found by Hakkila et al. (1994a), considering both isotropy and inhomogeneity. A smaller observing depth for a fixed number of observed GRBs would lead to a larger dipole moment. We find that a core radius of $R_{\text {core }}=23$ kpc would be typically found in 1005 locations by BATSE at the $99 \%$ confidence level. Hakkila et al. (1994a) found that the Dark Matter Halo can satisfy both the isotropy and inhomogeneity requirements of BATSE, however, a larger value of $R_{\text {core }}$ than $23 \mathrm{kpc}$ is required in order to avoid having too few faint GRBs.

In many halo models the nearest GRBs have a greater gradient and thus dipole moment than the entire distribution so the anisotropy might be first detectable in a subset of near GRBs. This is not the case for the Uniform Spherical Halo since if we do not view far enough to see its edge, the model becomes isotropic and homogeneous. For the Dark Matter Halo model, we find that for small datasets such that the total dataset limit on $R_{\text {core }}$ is $\lesssim R_{\circ}$, examining the brightest $1 / 4$ or $1 / 8$ of the GRBs gives a better limit. For larger datasets that already require a large value of $R_{\text {core }}$, examining a smaller dataset gives a less strict limit because the fluctuations of the smaller sample size are more important than the increased dipole moment. In any case, the brightest sources, e.g., datasets \#24, $25,31,32,38$, and 39 , are consistent with isotropy.

Smith (1994b) has proposed another geometric halo model, an exponential halo with source density $\propto \exp (-R / \bar{r})$. His example successful model has $\langle\cos \theta\rangle=0.056$, which deviates by $2.1 \sigma$ from the value observed for 1005 GRBs. This particular exponential halo is a fair match for the data. 


\subsection{High-Velocity Neutron Star Models}

The Dark Matter Halo model with a large core radius can match the isotropy and M31 constraints, and also the inhomogeneity constraint (Hakkila et al. 1994a). This model is primarily geometric because no candidate GRB source population has this distribution. This raises the question of whether the distribution of any known object can match the observations. Neutron stars are, of course, the most promising candidates.

Paczyński (1990) and Hartmann, Epstein \& Woosley (1990) calculated the orbits of neutron stars born in the disk. They found that if the distribution was viewed deeply enough to detect a deficiency of faint sources there would be a significant quadrupole moment $\left\langle\sin ^{2} b-\frac{1}{3}\right\rangle$. New observations and analyses since then have demonstrated the existence of a large number of high velocity neutron stars (e.g., Lyne \& Lorimer 1994; Frail, Goss, \& Whiteoak 1994). Lyne \& Lorimer (1994) find the mean neutron star velocity to be $450 \pm 90 \mathrm{~km} \mathrm{~s}^{-1}$ and the velocity distribution to be very broad, with an root-mean-square width of $525 \mathrm{~km} \mathrm{~s}^{-1}$. The mean velocity is close to the local speed to escape the Galaxy, $550 \pm 100 \mathrm{~km} \mathrm{~s}^{-1}$ (Fich \& Tremaine 1991). Many neutron stars are thus either unbound or only weakly bound, implying the existence of a much larger halo than formerly thought (Lyne \& Lorimer 1994). However, these observations still require a substantial fraction of the neutron stars to have velocities below the Galactic escape speed. The low-velocity neutron stars generate a disk population in addition to the halo formed by the high-velocity neutron stars, so this model still has difficulty matching the observations. A variety of solutions have been suggested: 1) a hypothetical population of neutron stars that are born in the halo; this population either being more numerous than that born in the disk or more efficient at bursting (Brainerd 1992; Eichler \& Silk 1992; Hartmann 1992; Fabian \& Podsiadlowski 1993), 2) a delayed turn-on so neutron stars still near the disk are not seen (Li \& Dermer 1992), 3) some mechanism such as a magnetic-field/recoil velocity correlation so that only high-velocity neutron stars produce gamma-ray bursts (Brainerd 1992; Li \& Dermer 1992), and 4) an alignment between the recoil velocity of a neutron star and its magnetically beamed gamma-ray burst emission (Li et al. 1994).

Eichler \& Silk (1992) propose that neutron stars are born in an extended halo via the mergers of Population III white dwarfs. They assume a Dark Matter Halo distribution with $R_{\text {core }}=40 \mathrm{kpc}$ and estimate $\langle\cos \theta\rangle$ to be 0.05 , which deviates from the observed value by about $1.8 \sigma$. Hartmann (1992) calculated the orbits of neutron stars born with 400 $\mathrm{km} \mathrm{s}^{-1}$ recoil velocities in an $R^{-2}$ halo of radius $50 \mathrm{kpc}$. An observing depth of $30 \mathrm{kpc}$ matches the intensity observations but yields $\left\langle\sin ^{2} b-\frac{1}{3}\right\rangle=-0.05,5.0 \sigma$ off from the current observations. Fabian \& Podsiadlowski (1993) propose that gamma-ray burst sources are

ejected from the Magellanic Clouds, producing an extended halo nearly homogeneous to 50 
kpc. Their example model has a dipole moment to the Magellanic Clouds of 0.038, which deviates by $1.9 \sigma$ from observed moment (Table 8 ). The agreement of these models with the data ranges from satisfactory to unacceptable.

Li \& Dermer (1992) proposed that gamma-ray bursts originate after a time delay from neutron stars born in the disk with velocities of $1000 \mathrm{~km} \mathrm{~s}^{-1}$. The time delay allows many of the sources to leave the disk before they become active, thereby reducing the disk contribution. Their model has $\langle\cos \theta\rangle=0.048$, which is $1.7 \sigma$ off from the current observed value.

The Halo Beaming Model of Li et al. (1994) assumes that 1) only high-velocity neutron stars emit GRBs, 2) these objects are born with their magnetic axis aligned with their recoil velocity, and 3) the gamma-ray burst emission is beamed in a $20^{\circ}$ half-angle beam along the magnetic axis. This beaming suppresses the observation of sources in the plane relative to those far above the plane. Li et al. (1994) determine the consequences of this model by following the orbits of neutron stars born in the disk with recoil velocities of 1000 $\mathrm{km} \mathrm{s}^{-1}$. This model still predicts a concentration in the plane, and, uniquely, a quadrupole concentration towards the Galactic center and anti-center; both concentrations should be most prominent for the nearest GRBs. The authors show, as a function of peak flux on the $1024 \mathrm{~ms}$ timescale, the model values of $\left\langle\sin ^{2} b-\frac{1}{3}\right\rangle$ and $\left\langle\cos ^{2} \theta-\frac{1}{3}\right\rangle$ for sets of all GRBs brighter than the peak flux value. The brightest $1 / 8$ of the first 1005 GRBs deviate from the model values by $2.0 \sigma$ and $3.1 \sigma$ (Table 8 ). This particular form of the Halo Beaming Model seems unlikely.

The models of Podsiadlowski et al. (1995) use several methods to reduce the anisotropy of neutron stars ejected from the plane. They follow the orbits of neutron stars in a non-spherical halo potential, which randomizes the orbits, thereby producing greater isotropy. In order to avoid a disk signal, they assume that only high-velocity neutron stars burst and that the sources have a delayed turn-on and a turn-off time. The latter requirement prevents the build of of a disk population formed by the capture of neutron stars that received their birth kick opposite to the Galactic rotation. Their example models (their Figures 5a and 5b) are based upon: 1) standard candle sources observed to $340 \mathrm{kpc}$, 2) neutron stars born with recoil velocities between 600 and $700 \mathrm{~km} \mathrm{~s}^{-1}$, 3) a turn-on delay of $10^{7}$ years, 4) turn-off times of $10^{9}$ years for model $5 \mathrm{a}$ and $10^{10}$ years for model $5 \mathrm{~b}$. The values of $\langle\cos \theta\rangle$ and $\left\langle\sin ^{2} b-\frac{1}{3}\right\rangle$ of their model $5 \mathrm{~b}$ are $2.0 \sigma$ and $2.2 \sigma$ off from those observed for $1005 \mathrm{GRBs}$, demonstrating that a source lifetime of $10^{10}$ years is probably too long. The moments of the $10^{9}$ year lifetime model are in good agreement with the data, deviating by $1.4 \sigma$ and $1.7 \sigma$. 


\subsection{Two-Component Models}

It has frequently been suggested that GRBs might originate from two populations in various combinations of disk, halo and cosmological distances (e.g., De Jager \& Schaefer 1992; Lingenfelter \& Higdon 1992; Smith \& Lamb 1993; Higdon \& Lingenfelter 1994; Katz 1994). Since sufficiently extended halo models agree with the observations we expect a small admixture of a disk component with either a halo or the isotropic distribution to also be satisfactory. As an example we consider a model with fraction $f_{\text {aniso }}$ of the locations drawn from the Watson distribution with $\kappa=-1$ and the remainder isotropic. The Watson distribution with $\kappa=-1$ has one-half of the probability within $b_{\frac{1}{2}}=23.1^{\circ}$ of the plane and $\left\langle\sin ^{2} b-\frac{1}{3}\right\rangle=-0.0796$, an $8.4 \sigma$ detection for 1000 GRBs; an example simulation is shown in Figure 1c. From Figure 8g we see that BATSE would typically detect at the $99 \%$ confidence level a 30\% contribution from this disk model in a sample of 1000 locations. Of course, the isotropy constraints would allow a greater fraction of a less concentrated disk component. However, the contribution of an almost isotropic component is limited by its near homogeneity.

Lingenfelter \& Higdon (1992) consider a model with two Galactic components arising from a single source population. The low-luminosity emissions are seen to a nominal distance of $300 \mathrm{pc}$ and the high-luminosity to $100 \mathrm{kpc}$. The source population is based upon the orbital calculations of Paczyński (1990) for Galactic neutron stars born in the disk. The sources seen at the great distances contribute most of the anisotropy - the low-luminosity sources are virtually isotropic and homogenous. This 1992 model now clearly disagrees with the data, differing from the observations for 1005 GRBs at the $3.5 \sigma$ level for $\langle\cos \theta\rangle$ and at the $6.1 \sigma$ level for $\left\langle\sin ^{2} b-\frac{1}{3}\right\rangle$. Their revised two-component model (Higdon \& Lingenfelter 1994) is formed from two geometric models: an exponential disk and a Dark Matter Halo model with a cutoff at $300 \mathrm{kpc}$. Since at most $25 \%$ of the GRBs are from the disk, the dominant anisotropies of their models are those of the halo portions, the dipole moment $\langle\cos \theta\rangle$. The comparisons are given in Table 8. The core radii of 7.5 and $15 \mathrm{kpc}$, which are "inferred from constraints on Galactic structure", are in very poor agreement with the observations. Even the model with a halo component with a core radius of $30 \mathrm{kpc}$ does not agree well with the observations. This last model has a disk component of only $8 \%$.

Smith \& Lamb (1993) consider several other disk plus halo models. Their disk model is an exponential sampled to $2 / 3$ of its scale height, resulting in a quadrupole moment $\left\langle\sin ^{2} b-\frac{1}{3}\right\rangle=-0.0401$, which is half the moment of our example two-component model (above, Figures 1c, 8g). One of their models has $20 \%$ of the sources from the disk, while the remainder are from a Dark Matter Halo with core radius $22.5 \mathrm{kpc}$ observed to $135 \mathrm{kpc}$. Again, this is predominantly a halo model, and it is currently $2.2 \sigma$ away from BATSE's 
measurement of $\langle\cos \theta\rangle$. Their other model has $67 \%$ of the GRBs originating in the disk and the remainder from a Gaussian shell halo model. The halo model is a Gaussian distribution with $\sigma=38 \mathrm{kpc}$ and its highest source density on a shell $25 \mathrm{kpc}$ from the Galactic center. Since this model is dominated by its disk component, the crucial statistic is $\left\langle\sin ^{2} b-\frac{1}{3}\right\rangle=$ -0.027 , which is $2.6 \sigma$ away from the value measured by BATSE for 1005 GRBs.

In this paper we are emphasizing the isotropy characteristics of two-component models. It is also necessary for models to match the observed peak flux distribution, $\log N-\log P$. A particular requirement for two-component models to agree with BATSE's observations is that the slope of the logarithmic cumulative peak flux distribution be concave down (Paczyński 1992; Smith \& Lamb 1993; Hakkila et al. 1994a; Harding 1994).

\subsection{Heliocentric Models}

No heliocentric pattern is evident $(\S 4)$. The lack of a dipole moment towards the Sun is a weak constraint: a spherical shell at the $19 \mathrm{AU}$ distance of Uranus would produce a dipole moment of $\langle\cos \phi\rangle=0.035$, which would be a $1.9 \sigma$ detection for 1005 GRBs (adapting eq. 1 from galactocentric to heliocentric shells). The above limit applies to the "typical" gamma-ray burst; testing the plane-wave nature of the wavefront of bright and presumably close bursts yields lower-limits of $\approx 40 \mathrm{AU}$ (Conners et al. 1993).

The models that have been suggested for a heliocentric origin involve comet-comet or comet-black hole collisions in the Oort cloud (Bickert \& Greiner 1993; Katz 1993; White 1993, Luchkov 1994). One can therefore constrain these models based upon our knowledge of the cometary cloud surrounding the Sun: models of the distribution of the heliocentric cometary cloud are based upon a solar system origin, planetary and stellar perturbations and the tidal force of the Galaxy (Weissman 1990; Clarke, Blaes \& Tremaine 1994). The Galactic tidal force has had negligible effect on the inner region of the cloud, which should thus have a strong ecliptic plane concentration reflecting its solar system origin (Clarke et al. 1994). The lack of an ecliptic plane concentration according to $\left\langle\sin ^{2} \beta-\frac{1}{3}\right\rangle$ is thus a useful constraint. In contrast, the distribution of the intermediate Oort cloud is dominated by Galactic tidal forces - Clarke et al. (1994) estimate $\left\langle\sin ^{2} b-\frac{1}{3}\right\rangle=+0.12$, which is quite inconsistent with the observed GRB locations. While the distant portion of the cloud is probably more isotropic, producing GRBs there but not in the inner portion is very implausible since the cometary density is lower and the comets are more distant (Clarke et al. 1994).

A severe problem for Oort cloud models of GRBs is the homogeneity of the brightest 
GRBs (Maoz 1993b; Clarke et al. 1994; Horack et al. 1994), which has been well-observed by instruments with greater exposure than BATSE (e.g., Fenimore et al. 1993). The physics of the models pose even greater problems (Clarke et al. 1994).

\section{Discussion}

If gamma-ray bursts have a large-scale anisotropy, it is remarkably small, especially compared to any observed Galactic component. The most extended, observed component of the Galaxy is the halo, as traced by stars or globular clusters. The globular clusters are distributed in galactocentric radius $R$ as $R^{-3.5}$ (Zinn 1985; Gilmore, King \& van der Kruit 1990; Djorgovski \& Meylan 1994). This contrasts with the more isotropic $R^{-2}$ we used in the Dark Matter Halo form (eq. 2). Using a slightly different halo form than ours, Djorgovski \& Meylan (1994) find a core radius of at most $2 \mathrm{kpc}$ for the globular clusters, which contrasts with the $2 \sigma$ lower limit for the core radius of the GRBs of $11 \mathrm{kpc}$. Zinn (1985) has shown that the globular clusters consist of two populations, a thick disk and a halo, and that population membership is reliably determined by cluster metallicity. The 93 globular clusters identified by Zinn (1985) as halo members have $\langle\cos \theta\rangle=0.617$ and $\left\langle\sin ^{2}-\frac{1}{3}\right\rangle=$ -0.111 , which are, respectively, $10.3 \sigma$ and $3.6 \sigma$ deviations from isotropy! Selection effects against globular clusters near the Galactic plane or center make the observed distribution more isotropic than the true distribution (Zinn 1985, Djorgovski \& Meylan 1994). These moments contrast sharply with those of the first 1005 GRBs observed by BATSE: the $2 \sigma$ limits corrected for sky exposure bias are $\langle\cos \theta\rangle<0.053$ and $\left\langle\sin ^{2}-\frac{1}{3}\right\rangle>-0.022$.

While geometric Galactic models for the distribution of GRBs can match the observed isotropy, they are different from any known Galactic distribution. Although there is observational and theoretical support for an exponential disk, this is not the case for an exponential halo (Gilmore, King \& van der Kruit 1990). We know of no Galactic component with its peak density $25 \mathrm{kpc}$ from the Galactic center, as is assumed in the Gaussian shell halo model. The maximum core radius for the Dark Matter Halo component of the Galaxy models of Caldwell \& Ostriker (1981) is about $9 \mathrm{kpc}$. While the $2 \sigma$ upper limit for $\langle\cos \theta\rangle$ implies a core radius of at least $11 \mathrm{kpc}$, taking account of the GRB peak flux distribution demands a much larger core radius (Hakkila et al. 1994a). The model of Higdon \& Lingenfelter (1994) with a core radius of $15 \mathrm{kpc}$ has a value of $\langle\cos \theta\rangle$ which is $3.1 \sigma$ in excess of the value observed for 1005 GRBs (Table 8). While previously core radii consistent with the Dark Matter Halo form as used to explain the rotation curve of the Milky Way were consistent with BATSE's GRB observations, this is no longer true. Our geometric Dark Matter Halo assumed spherical symmetry, whereas Dark Matter Halos are 
actually expected to be quite flat (Dubinski \& Carlberg 1991).

Possible physical bases for the Dark Matter Halo distribution for GRBs are that the $R^{-2}$ dependence is similar to that of an isothermal halo (Gilmore, King \& van der Kruit 1990) and to the density of very-high velocity objects ejected from the Galaxy. Many recent models have been based upon the assumption that the sources of GRBs are neutron stars born with velocities comparable to or greater than the escape speed from the Galaxy. The mechanism postulated to cause only high-velocity neutron stars to burst must be very effective. The fraction of observed GRBs originating from low-velocity neutron stars, which are confined to a thick disk, must be small (§5.7). The fraction of GRB sources which have low velocities must be yet smaller since many of the high-velocity neutron stars are unbound and escape the observable region, which must have a radius $\lesssim 350 \mathrm{kpc}$ because of the M31 constraint. Even the assumption that only high-velocity neutron stars produce GRBs is insufficient to match the data - all of the models have at least one additional assumption: 1) a delayed turn-on (Li \& Dermer 1992; Podsiadlowski et al. 1995), 2) a turn-off time (Podsiadlowski et al. 1995), 3) a population of high-velocity neutron stars born in the halo which dominate neutron stars born in the disk as GRB sources (Eichler \& Silk 1992; Hartmann 1992), 4) a preferential production of GRB sources in the Magellanic Clouds (Fabian \& Podsiadlowski 1993), and 5) an alignment between the gamma-ray emission and the recoil velocity of the neutron star (Li et al. 1994).

Since a very-extended halo is consistent with the data, it is possible to have twocomponent models with one or two Galactic components. The observations currently require that at most a small fraction of the bursts originate from a disk component (§5.7). A common motivation for two population models is allow GRBs with cyclotron lines to originate from disk neutron stars in the solar neighborhood. Models of photon emission from magnetic neutron stars were developed when it was generally believed that the distance to GRBs was of order 100 pc. It seems unlikely that these models will work at halo distances which are $\gtrsim 100$ times greater. Two population models generally have a ratio of the distances of the populations of at least 100, implying a ratio of luminosities of order $10^{4}$ or more. It seems implausible that phenomena with such greatly different luminosities would not be observationally separable into two classes. The observed bimodality in GRB durations (Mazets et al. 1981; Hurley 1991; Klebesadel 1992; Dezalay et al. 1992; Kouveliotou et al. 1993b) is the best indication for two possible classes, but both the short and long GRBs are consistent with isotropy (datasets \#14 and \#15) and inconsistent with homogeneity (Kouveliotou et al. 1993b). Invoking two populations to explain the GRB position and intensity distributions would seem to double the difficulty of explaining the physics of gamma-ray bursts. 
Several of the Galactic models have moments in acceptable agreement with the data - the best has a moment $1.7 \sigma$ in excess of the observations (Table 8). However, most of the Galactic models created in response to BATSE's observations have moments that deviate by two or more $\sigma$ from the data. While if GRBs are Galactic, only the true model need predict moments consist with the observations, the failure of the majority of the post-BATSE models demonstrates the difficulty of matching Galactic models to BATSE's observations. Each model has been tested by comparing the observations with the model characteristics for at most one dipole and one quadrupole moment. Further tests of the models are possible, such as comparing their radial distributions with the observed $\log N-\log P$ distribution.

The simultaneous observations by BATSE of the isotropy and the strong inhomogeneity of GRBs disprove the previous paradigm of a Galactic disk origin. No known Galactic component, including the Dark Matter halo, is sufficiently isotropic to match the observational constraints. Consequently, current models either postulate a new Galactic component or extreme energy requirements for sources at cosmological distances. If GRBs are Galactic, the lack of excess GRBs from the Galactic Center and M31 constrain the distance of the typical GRB observed by BATSE to between 100 and $300 \mathrm{kpc}(\S 5.5)$. In contrast to the difficulty that the isotropy observations pose for Galactic models, isotropy is a natural consequence of an origin at cosmological distances. The observed large-scale isotropy strongly favors but does not require that gamma-ray bursts originate at cosmological distances. The continued observation of gamma-ray bursts by BATSE promises to either detect an anisotropy or to further tighten the constraints on Galactic models.

M. S. Briggs acknowledges support provided by NASA through the Compton GRO Fellowship Program, grant GRO/PFP-91-06. D. Hartmann acknowledges support from NASA grant NAG-51578. We thank J. J. Brainerd, D. Lamb and the referees, J. Higdon and P. Podsiadlowski, for their useful suggestions, and the BATSE operations team for their efforts. 


\section{REFERENCES}

Atteia, J.-L., \& Hurley, K. 1986, Adv. Space Res., 6, \#4, 39

Atteia, J.-L., \& Dezalay, J.-P. 1993, A\&A, 274, L1

Best, D. J., \& Fisher, N. I. 1986, Austral. J. Statist., 28, 13

Bickert, K. F., \& Greiner, J. 1993, in AIP Conf. Proc. 280: Compton Gamma-Ray

Observatory, ed. M. Friedlander, N. Gehrels, D. J. Macomb, (New York: AIP), 1059

Bingham, C. 1974 Ann. Stat., 2, 1201

Brainerd, J. J. 1992, Nature, 355, 522

Brainerd, J. J. 1994, ApJ, 428, 21

Brainerd, J. J, 1995, ApJ, in press

Brainerd, J. J., Meegan, C. A., Briggs, M. S., Pendleton, G. N., Brock, M. N. 1995, ApJ, 441, L39

Briggs, M. S. 1993, ApJ, 407, 126

Briggs, M. S., Paciesas, W. S., Brock, M. N., Fishman, G. J., Meegan, C. A., \& Wilson, R. B. 1993a, in AIP Conf. Proc. 280: Compton Gamma-Ray Observatory, ed. M. Friedlander, N. Gehrels, D. J. Macomb, (New York: AIP), 686

Briggs, M. S., Paciesas, W. S., Brock, M. N., Fishman, G. J., Meegan, C. A., \& Wilson, R. B. 1993b, in AIP Conf. Proc. 280: Compton Gamma-Ray Observatory, ed. M. Friedlander, N. Gehrels, D. J. Macomb, (New York: AIP), 691

Briggs, M. S., Paciesas, W. S., Pendleton, G. N., Fishman, G. J., Meegan, C. A., \& Wilson, R. B. 1994, in AIP Conf. Proc. 307: Gamma-Ray Bursts, ed. G. J. Fishman, J. J. Brainerd, K. Hurley, (New York: AIP), 44

Brock, M. N., Meegan, C. A., Roberts, F. E., Fishman, G. J., Wilson, R. B., Paciesas, W. S., \& Pendleton, G. N. 1992a, in AIP Conf. Proc. 265: Gamma-Ray Bursts, ed. W. Paciesas and G. J. Fishman, (New York: AIP), 383

Brock, M. N., Meegan, C. A., Fishman, G. J., Wilson, R. B., Paciesas, W. S. \& Pendleton, G. N. 1992b, in AIP Conf. Proc. 265: Gamma-Ray Bursts, ed. W. Paciesas and G. J. Fishman, (New York: AIP), 399

Caldwell, J. A. R., \& Ostriker, J. P. 1981, ApJ, 251, 61

Clarke, T. E., Blaes, O., \& Tremaine, S. 1994, AJ, 107, 1873

Conners, A., et al. 1993, A\&AS, 97, 75 
De Jager, O. C., \& Schaefer, B. E. 1992, in AIP Conf. Proc. 265: Gamma-Ray Bursts, ed. W. Paciesas and G. J. Fishman, (New York: AIP), 226

Dermer, C. 1992, Phys. Rev. Lett., 68, 1799

Dermer, C. D., \& Schlickeiser, R. 1994, in AIP Conf. Proc. 307: Gamma-Ray Bursts, ed. G. J. Fishman, J. J. Brainerd, K. Hurley, (New York: AIP), 510

Dezalay, J.-P., Barat, C., Talon, R., Sunyaev, R., Terekhov, O., \& Kuznetsov, A. 1992, in AIP Conf. Proc. 265: Gamma-Ray Bursts, ed. W. Paciesas and G. J. Fishman, (New York: AIP), 304

Djorgovski, S., \& Meylan, G. 1994, AJ, 108, 1292

Dubinski, J., \& Carlberg, R. G. 1991, ApJ, 378, 496

Eadie, W. T., Drijard, D., James, F. E., Roos, M., \& Sadoulet, B. 1971, Statistical Methods in Experimental Physics, (Amsterdam: North-Holland)

Eichler, D., \& Silk, J. 1992, Science, 257, 937

Fabian, A. C., \& Podsiadlowski, P. 1993, MNRAS, 263, 49

Fenimore, E. E., Epstein, R. I., Ho, C., Klebesadel, R. W., Lacey, C., Laros, J. G., Meier, M., Strohmayer, T., Pendleton, G., Fishman, G., Kouveliotou, C., \& Meegan, C. 1993, Nature, 366, 40

Fich, M., \& Tremaine, S. 1991, ARA\&A, 29, 409

Fisher, N. I., Lewis, T., \& Embleton, B. J. J. 1987, Statistical Analysis of Spherical Data, (Cambridge: Cambridge Univ. Press)

Fishman, G. J. 1979, ApJ, 233, 851

Fishman, G. J., Meegan, C. A., Wilson, R. B., Brock, M. N., Horack, J. M., Kouveliotou, C., Howard, S., Paciesas, W. S., Briggs, M. S., Pendleton, G. N., Koshut, T. M., Mallozzi, R. S., Stollberg, M., \& Lestrade, J. P. 1994, ApJS, 92, 229

Frail, D. A., Goss, W. M., \& Whiteoak, J. B. Z. 1994, ApJ, 437, 781

Gilmore, G. F., King, I. R., \& van der Kruit, P. C. 1990, The Milky Way as a Galaxy, (Mill Valley, CA: Univ. Science Books)

Graziani, C. 1993, private communication

Gurevich, A. V., Zharkov, G. F., Zybin, K. P., \& Ptitsyn, M. O. 1994, Phys. Lett. A, 192, 305

Hakkila, J., Meegan, C. A., Pendleton, G. N., Fishman, G. J., Wilson, R. B., Paciesas, W. S., Brock, M. N., \& Horack, J. M. 1994a, ApJ, 422, 659 
Hakkila, J., Meegan, C. A., Fishman, G. J., Wilson, R. B., Brock, M. N., Horack, J. M., Pendleton, G. N., Paciesas, \& Briggs, M. S. 1994b, in AIP Conf. Proc. 307: Gamma-Ray Bursts, ed. G. J. Fishman, J. J. Brainerd, K. Hurley, (New York: AIP), 59

Harding, A. K. 1994, ApJS, 90, 863

Hartmann, D., \& Epstein, R. I. 1989, ApJ, 346, 960

Hartmann, D., Epstein, R., \& Woosley, S. E. 1990, ApJ, 348, 625

Hartmann, D. 1992, Comments Astrophys., 16, 231

Hartmann, D., Brown, L. E., The, L.-S., Linder, E. V., Petrosian, V., Blumenthal, G., \& Hurley, K. C. 1994a, ApJS, 90, 893

Hartmann, D., Blumenthal, G. R., Briggs, M. S., Fishman, G. J., Hakkila, J., Hurley, K., Horack, J., Kouveliotou, C., Meegan, C. A., Paciesas, W. S., Pendleton, G. N. 1994b, ApJ, submitted

Higdon, J. C., \& Lingenfelter, R. E. 1990, ARA\&A, 28, 401

Higdon, J. C., \& Lingenfelter, R. E. 1994, ApJ, 434, 552

Horack, J. M. 1991, NASA Reference Pub. 1268: Development of the Burst and Transient Source Experiment (BATSE), (Huntsville, AL: NASA/MSFC)

Horack, J. M., Meegan, C. A., Fishman, G. J., Wilson, R. B., Paciesas, W. S., Emslie, A. G., Pendleton, G. N., \& Brock, M. N., 1993, ApJ, 413, 293

Horack, J. M., \& Emslie, A. G. 1994, ApJ, 428, 620

Horack, J. M., Koshut, T. M., Mallozzi, R. S., Storey, S. D., \& Emslie, A. G. 1994, ApJ, 429, 319

Hurley, K., editor 1986, in AIP Conf. Proc. 141: Gamma-Ray Bursts, ed. E. P. Liang, \& V. Petrosian, (New York: AIP), 1

Hurley, K. 1991, in AIP Conf. Proc. 265: Gamma-Ray Bursts, ed. W. S. Paciesas \& G. J. Fishman, (New York: AIP), 3

Hurley, K. 1993, private communication

Hurley, K., Sommer, M., Kouveliotou, C., Fishman, G., Meegan, C., Cline, T., Boer, M., \& Niel, M. 1994, in AIP Conf. Proc. 307: Gamma-Ray Bursts, ed. G. J. Fishman, J. J. Brainerd, K. Hurley, (New York: AIP), 27

Jennings, M. C. 1982, ApJ, 258, 110

Katz, J. I. 1993, in AIP Conf. Proc. 280: Compton Gamma-Ray Observatory, ed. M. Friedlander, N. Gehrels, D. J. Macomb, (New York: AIP), 1090 
Katz, J. I. 1994, ApJ, 422, 248

Klebesadel, R. W. 1992, in Gamma-Ray Bursts, ed. C. Ho, R. I. Epstein \& E. E. Fenimore, (Cambridge: Cambridge Univ. Press), 161

Koshut, T. M., Paciesas, W., Pendleton, G., Brock, M. N., Fishman, G. J., Meegan, C. A., \& Wilson, R. B. 1994, in AIP Conf. Proc. 307: Gamma-Ray Bursts, ed. G. J. Fishman, J. J. Brainerd, K. Hurley, (New York: AIP), 697

Kouveliotou, C., Fishman, G. J., Meegan, C. A., Paciesas, W. S., Wilson, R. B., van Paradijs, J., Preece, R. D., Briggs, M. S., Pendleton, G. N., Brock, M. N., Koshut, T. M., \& Horack, J. M. 1993a, Nature, 362, 728

Kouveliotou, C., Meegan, C. A., Fishman, G. J., Bhat, N. P., Briggs, M. S., Koshut, T. M., Paciesas, W. S., \& Pendleton, G. N. 1993b, ApJ, 413, L101

Kouveliotou, C., Fishman, G. J., Meegan, C. A., Paciesas, W. S., van Paradijs, J., Norris, J. P., Preece, R. D., Briggs, M. S., Horack, J. M., Pendleton, G. N., \& Green, D. A. 1994a, Nature, 368, 125

Kouveliotou, C. 1994b, Nature, 370, 26

Lamb, D. Q., Graziani, C., \& Smith, I. A. 1993, ApJ, 413, L11

Li, H., \& Dermer, C. D. 1992, Nature, 359, 514

Li, H., Duncan, R., \& Thompson, C. 1994, in AIP Conf. Proc. 307: Gamma-Ray Bursts, ed. G. J. Fishman, J. J. Brainerd, K. Hurley, (New York: AIP), 600

Li, H. 1995, private communication

Liang, E. P., \& Li, H. 1993, A\&A, 273, L53

Lingenfelter, R. E., \& Higdon, J. C. 1992, Nature, 356, 132

Lyons, L. 1986, Statistics for Nuclear and Particle Physicist, (Cambridge: Cambridge Univ. Press)

Lyne, A. G., \& Lorimer, D. R. 1994, Nature, 369, 127

Luchkov, B. I. 1994, Astron. Letters, 20, 253

Mallozzi, R. S., Paciesas, W. S., Meegan, C. A., Fishman, G. J., \& Wilson, R. B. 1993, in AIP Conf. Proc. 280: Compton Gamma-Ray Observatory, ed. M. Friedlander, N. Gehrels, D. J. Macomb, (New York: AIP), 1122

Mao, S., \& Paczyński, B. 1992a, ApJ, 388, L45

Mao, S., \& Paczyński, B. 1992b, ApJ, 389, L13

Maoz, E. 1993a, Harvard-Smithsonian CfA preprint \#3613 
Maoz, E. 1993b, ApJ, 414, 877

Martin, B. R. 1971, Statistics for Physicists, (London: Academic)

Mazets, E. P., Golenetskii, S. V., Il'inskii, V. N., Panov, V. N., Aptekar, R. L., Gur'yan, Yu. A., Proskura, M. P., Sokolov, I. A., Sokolova, Z. Ya., Kharitonova, T. V., Dyatchkov, A. V., \& Khavenson, N. G. 1981, Ap\&SS, 80, 3

McBreen, B., \& Metcalfe, L. 1988, Nature, 332, 234

McBreen, B., Plunkett, S., \& Metcalfe, S. 1993, A\&AS, 97, 81

Meegan, C. A., Fishman, G. J., Wilson, R. B., Paciesas, W. S., Brock, M. N., Horack, J. M., Pendleton, G. N., \& Kouveliotou, C. 1991, IAU Circ. 5358

Meegan, C. A., Fishman, G. J., Wilson, R. B., Paciesas, W. S., Pendleton, G. N., Horack, J. M., Brock, M. N., \& Kouveliotou, C. 1992a, Nature, 355, 143

Meegan, C. A., Fishman, G. J., Wilson, R. B., Paciesas, W. S., Brock, M. N., Horack, J. M., Pendleton, G. N., \& Kouveliotou, C. 1992b, IAU Circ. 5641

Meegan, C. A., Fishman, G., Wilson, R., Brock, M., Horack, J., Paciesas, W., Pendleton, G., \& Kouveliotou, C. 1993a, in AIP Conf. Proc. 280: Compton Gamma-Ray Observatory, ed. M. Friedlander, N. Gehrels, D. J. Macomb, (New York: AIP), 681

Meegan, C. A., Fishman, G., Wilson, R., \& Paciesas, W. 1993b, in AIP Conf. Proc. 280: Compton Gamma-Ray Observatory, ed. M. Friedlander, N. Gehrels, D. J. Macomb, (New York: AIP), 1117

Meegan, C. A., Fishman, G., Wilson, R., Brock, M., Horack, J., Paciesas, W., Pendleton, G., Briggs, M., Koshut, T., Kouveliotou, C., Teegarden, B., Matteson, J., \& Hakkila, J. 1994a, in AIP Conf. Proc. 307: Gamma-Ray Bursts, ed. G. J. Fishman, J. J. Brainerd, K. Hurley, (New York: AIP), 3

Meegan, C. A., Fishman, G. J., Horack, J. M., Brock, M. N., Cole, S., Paciesas, W. S., Briggs, M. S., Pendleton, G. N., Preece, R., Koshut, T. M., Mallozzi, R. S., Kouveliotou, C., \& McCollough, M. 1994b, electronic catalog available via telnet grossc.gsfc.nasa.gov, username gronews

Meegan, C. A., Hartmann, D. H., Brainerd, J. J., Briggs, M. S., Paciesas, W. S., Pendleton, G. N., Kouveliotou, C., Fishman, G., Blumenthal, G. \& Brock, M. 1995, ApJ, 446, L15

Paczyński, B. 1986, ApJ, 308, L43

Paczyński, B. 1990, ApJ, 348, 485

Paczyński, B. 1991a, Acta Astron., 41, 257 
Paczyński, B. 1991b, Acta Astron., 41, 157

Paczyński, B. 1992, Acta Astron., 42, 1

Paczyński , B., \& Xu, G. 1994, ApJ, 427, 708

Pendleton, G. N., Mallozzi, R. S., Paciesas, W. S., Briggs, M. S., Preece, R. D., Koshut, T. M., Horack, J. M., Meegan, C. A., Fishman, G. J., Hakkila, J., \& Kouveliotou, C. 1995, ApJ, submitted

Piran, T. 1992, ApJ, 389, L45

Podsiadlowski, P., Rees, M. J., \& Ruderman, M. 1995, MNRAS, 273, 755

Prilutski, O. F., \& Usov, V. V. 1975, Ap\&SS, 34, 395

Quashnock, J. M., \& Lamb, D. Q. 1993a, MNRAS, 265, L45

Quashnock, J. M., \& Lamb, D. Q. 1993b, MNRAS, 265, L59

Quashnock, J. M. 1995, private communication

Shklovskii, I. S., \& Mitrofanov, I. G. 1985, MNRAS, 212, 545

Smith, I. A., \& Lamb, D. Q. 1993, ApJ, 410, L23

Smith, I. A. 1994a ApJ, 429, L65

Smith, I. A. 1994b, in AIP Conf. Proc. 307: Gamma-Ray Bursts, ed. G. J. Fishman, J. J. Brainerd, K. Hurley, (New York: AIP), 103

Usov, V. V., \& Chibisov, G. V. 1975, Soviet Ast., 19, 115

van den Bergh, S. 1983, Ap\&SS, 97, 385

Wang, V. C., \& Lingenfelter, R. E. 1993, ApJ, 416, L13

Wang, V. C., \& Lingenfelter, R. E. 1995, ApJ, 441, 747

Wasserman, I. 1992, ApJ, 394, 565

Watson, G. S. 1983, Statistics on Sphere, (New York: John Wiley \& Sons)

Weissman, P. R. 1990, Nature, 344, 825

White, R. S. 1993, Ap\&SS, 208, 301

Wickramasinghe, W. A. D. T., Nemiroff, R. J., Norris, J. P., Kouveliotou, C., Fishman, G. J., Meegan, C. A., Wilson, R. B., \& Paciesas, W. S. 1993, ApJ, 411, L55

Woods, E., \& Loeb, A. 1994, ApJ, 425, L63

Zinn, R. 1985, ApJ, 293, 424 
Table 2. ANGULAR ERRORS: DATASET \#6 AND SIMULATIONS

\begin{tabular}{ccccc}
\hline \hline \multicolumn{3}{c}{$2 \mathrm{~B}$ less MAXBC } & \multicolumn{2}{c}{ Simulations } \\
Fraction & $\begin{array}{c}\sigma_{\text {stat }} \\
(\text { degrees })\end{array}$ & $\begin{array}{c}\sigma_{\text {tot }} \\
\text { (degrees) }\end{array}$ & Fraction & $\begin{array}{c}\Sigma \\
\text { (degrees) }\end{array}$ \\
\hline $50 \%$ & $0.1-3.7$ & $4.0-5.4$ & $45 \%$ & 15. \\
$25 \%$ & $3.7-7.4$ & $5.4-8.4$ & $22.5 \%$ & 20. \\
$18.8 \%$ & $7.4-13.8$ & $8.4-14.4$ & $16.875 \%$ & 30. \\
$6.2 \%$ & $13.9-29.9$ & $14.5-30.1$ & $15.625 \%$ & 60. \\
\hline
\end{tabular}


Table 3. EXPECTED VALUES OF THE STATISTICS: CORRECTED FOR BATSE'S SKY EXPOSURE

\begin{tabular}{ccc}
\hline \hline Statistic & Mean $^{\mathrm{a}, \mathrm{b}}$ & $\sigma$ \\
\hline$\langle\cos \theta\rangle$ & -0.013 & $0.99 \sqrt{\frac{1}{3 N_{B}}}$ \\
$\left\langle\sin ^{2} b-\frac{1}{3}\right\rangle$ & -0.005 & $0.99 \sqrt{\frac{4}{45 N_{B}}}$ \\
$\mathcal{W}$ & $3+0.0020 N_{B}$ & $\ldots$ c \\
$\mathcal{B}$ & $5+0.0077 N_{B}$ & $\ldots . \mathrm{c}$ \\
$\langle\sin \delta\rangle$ & 0.026 & $1.04 \sqrt{\frac{1}{3 N_{B}}}$ \\
$\left\langle\sin ^{2} \delta-\frac{1}{3}\right\rangle$ & 0.026 & $1.03 \sqrt{\frac{4}{45 N_{B}}}$ \\
$\langle\cos \phi\rangle$ & $0.010 \cos \left[\frac{2 \pi(\mathrm{T}-9160)}{365.25}\right]$ & $0.98 \sqrt{\frac{1}{3 N_{B}}}$ \\
$\left\langle\sin ^{2} \beta-\frac{1}{3}\right\rangle$ & 0.020 & $1.02 \sqrt{\frac{4}{45 N_{B}}}$ \\
\hline
\end{tabular}

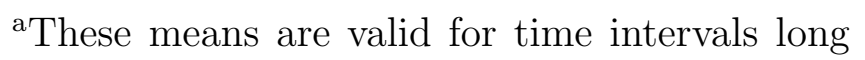
enough to average over the CGRO precession period of about 50 days.

${ }^{\mathrm{b}} T$ is the time in Truncated Julian Day Number (TJD), which is the Julian Day Number minus 2440000.5

${ }^{\mathrm{c}}$ see Figure 4. 
Table 5. RESULTS: EARTH COORDINATE SYSTEM TESTS

\begin{tabular}{|c|c|c|c|c|c|c|c|c|}
\hline \multicolumn{3}{|c|}{ Dataset } & \multicolumn{3}{|c|}{$\langle\sin \delta\rangle$} & \multicolumn{3}{|c|}{$\left\langle\sin ^{2} \delta-\frac{1}{3}\right\rangle$} \\
\hline \# & Name & Size & Value & Dev. $^{a}$ & Raw Dev. & Value & Dev. $^{a}$ & Raw Dev. \\
\hline 2 & $1 \mathrm{~B}$ revised & 262 & 0.015 & -0.3 & +0.4 & 0.000 & -1.3 & 0.0 \\
\hline 3 & $2 \mathrm{~B}$ & 585 & 0.003 & -0.9 & +0.1 & 0.024 & -0.1 & +2.0 \\
\hline 1 & first 1005 & 1005 & 0.016 & -0.5 & +0.9 & 0.019 & -0.7 & +2.0 \\
\hline
\end{tabular}

${ }^{\text {a }}$ Deviation, in $\sigma$, of the observed value from the value calculated from BATSE's sky exposure map assuming isotropy.

b Deviation, in $\sigma$, of the observed value from the value expected for isotropy ignoring BATSE's sky exposure. 